\title{
Analytical Solution to Heat Transfer in Compressible Laminar Flow \\ in a Flat Minichannel
}

\author{
Cheng Bao ${ }^{\mathrm{a} *}$, Zeyi Jiang ${ }^{\mathrm{a}}$, Xinxin Zhang ${ }^{\mathrm{a}}$, John T.S. Irvine ${ }^{\mathrm{b}}$ \\ a. School of Energy and Environmental Engineering, University of Science \& Technology Beijing, Beijing, 100083, \\ P.R. China \\ b. School of Chemistry, University of St Andrews, St Andrews, Fife KY16 9ST, Scotland, UK.
}

\begin{abstract}
:
Heat transfer in compressible laminar flow in mini-/micro-channels, a classical and general topic in fields of fuel cells, electronics, micro heat exchanger, etc., is revisited. Based on a two-dimensional continuum flow model, analytical solutions of the dimensionless model are achieved in closed-form symbolic algebras of Whittaker eigenfunctions, corresponding to two kinds of boundary conditions with arbitrarily prescribed wall temperature or wall heat flux. As the eigenvalues and eigenfunctions are independent on the dimensionless quantities, which influence the along-the-channel behaviors, the algorithm reveals the common features of compressible laminar thermal flows. The algorithms do not require the assumption of a linear pressure distribution, which is proved to be untenable in some cases (e.g. constant wall heat flux). The algorithms are validated well by the exact (numerical) computations in exemplary cases of both small and moderate Reynolds number, Mach number and Eckert number of air. Any Prandtl number of the fluid is applicable.
\end{abstract}

Key words: Minichannel; heat transfer; compressible laminar flow; analytical solution; Whittaker function

\section{Introduction}

With hydraulic diameters ranging from microns to millimeters, and correspondingly outstanding heat transfer performance due to the large specific surface area, minichannels and microchannels have been being increasingly used in many different applications, e.g. gas distributors or flow field plates in fuel cells, compact heat exchangers, heat sinks for electronics cooling, temperature control in injection molding of plastic or composite materials, micro heat pipes in micro cooling system, and on-chip laboratories, etc. [1]

Fluid flows and heat transfer in mini- and microchannels have been investigated a lot [1-5]. Besides large amounts of experimental studies and numerical calculations, analytical studies were performed to understand the fundamentals, including prediction of thermo-hydraulic performance, analysis of stability, parameter estimation in heat-transfer inverse problems, and also help in reducing the numerical computational cost. Brinkman [6] early calculated the temperature distribution in a capillary accounting for the energy dissipation of viscous flow under the conditions of a uniform wall heat flux or a homogeneous wall temperature. With an analytical solution in form of a series expansion, Siegel et al. [7] analyzed the heat transfer characteristics for a laminar forced convection flow in a circular tube with prescribed, i.e. uniform and arbitrary longitudinal variation of wall heat flux. Barron et al. [8] extended the Graetz problem, i.e. thermally developing heat transfer in laminar flow through a circular tube, to slip-flow regime accounting for the velocity slip

\footnotetext{
${ }^{*}$ Corresponding author. Tel: +86-10-62333682, Fax: +86-10-62329145

E-mail: baocheng@mail.tsinghua.edu.cn (C. Bao)
} 
and the temperature jump boundary conditions, and solved the eigenfunctions by the power-series method. With the similar mathematical method, for a gaseous flow in two-dimensional micro and nano-channels, Hadjiconstantinou and Simek [9] investigated the constant wall-temperature convective heat-transfer characteristics in the slip-flow regime $0 \leq K n \leq 0.2$, where $K n$ the Knudsen number, defined as the ratio between the molecular mean free path and the channel height. They found that it is necessary to include the effects of axial heat conduction in the continuum model due to the finite Peclet numbers. Rouizi et al. [10] presented an analytical solution of conjugate heat transfer in flat mini-channel based on Fourier transform of the temperature and normal flux, and then used it inversely to estimate the fluid bulk temperature distribution from the external surface heat sources and the corresponding noised temperature profiles. Among these studies, incompressible fluids were considered, also for gaseous flow because of the small pressure drop and the small Mach number of flow in mini- and microchannels. For compressible flows, Prud'homme et al. [11] early presented an approximate solution for the expansion cooling of a compressible idealgas fluid, with a double perturbation expansion of the radius to length ratio and the relative pressure drop. Vandenberg et al. [12] analytically investigated the velocity distribution of a steady, isothermal, compressible and laminar flow in a capillary, with a perturbation expansion of compressibility of the fluid. By setting the fluid inlet temperature equal to the homogenous wall temperature, Vandenberg et al. [13] then analytically investigated the thermal effects (expansion cooling and dissipation heating) of a compressible viscous flow. In this work, the eigenfunctions of the temperature distribution were expressed in form of power series, and the polynomial coefficients were numerically obtained from a set of linear equations. Harley et al. [14] made a theoretical investigation of compressible ideal-gas flow with low Reynolds number and high Mach number in microchannels, and presented a one-dimensional analytical solution of the axial distribution of the gas density for an isothermal problem. Schlichting and Gersten [15] summarized the boundary-layer theory of incompressible and compressible flow, among the algorithms only some specific cases have the analytical solutions in closed form for compressible thermal flow.

In this article, we revisit the compressible gaseous thermal flow in a flat minichannel. The contents of the article are organized to first introduce the hydrodynamic equations, which are subsequently reduced and normalized based on scale analysis and dimensionless quantities. Then analytical solutions of the two-dimensional model are performed in closed-form symbolic algebras of eigenfunctions. Both the cases of boundary conditions of arbitrarily prescribed wall temperature and wall heat flux are included.

\section{Mathematical model}

For our problem, i.e. a parallel-plate channel as shown in Figure 1, an uniform flow from infinity is first isothermally fully developed to obtain a parabola velocity profile, then within the range of $x$ $=0$ and $x=L$ the fluid has heat exchange with the walls with prescribed wall temperature or wall heat flux. The following assumptions are made:

(a) The continuum hypothesis is applicable and there is no slip flow at the wall.

(b) The flow is steady, laminar, with negligible volume forces and no external heat sources.

(c) The fluid is Newtonian with constant dynamic viscosity $(\mu)$, constant-pressure specific heat $\left(c_{p}\right)$ and thermal conductivity $(\kappa)$,

(d) $y$-coordinate velocity $(v)$ is safely discarded, i.e. $v=0$. 


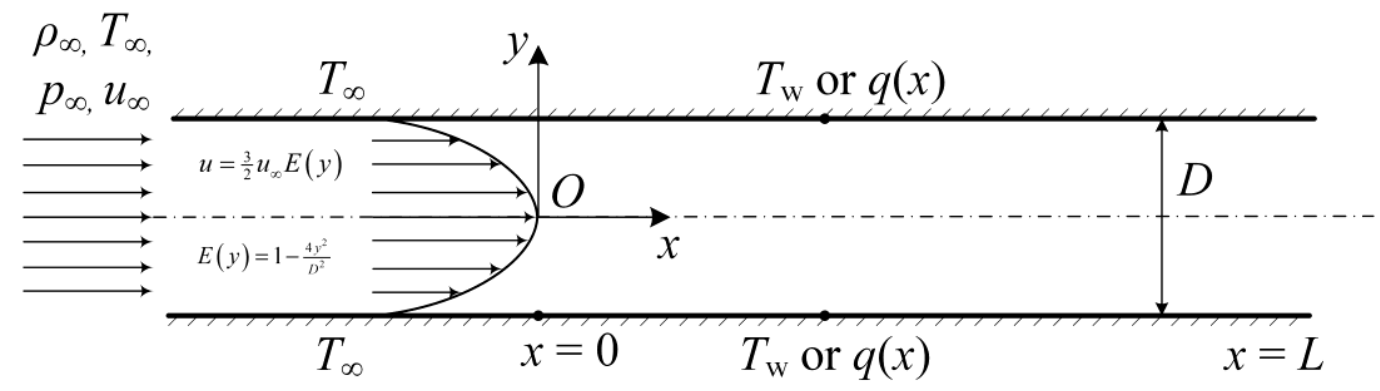

Fig. 1 Geometry of a flat minichannel.

The hydrodynamic equations for conversation of mass, momentum and energy in the twodimensional Cartesian coordinate system are

$$
\begin{gathered}
\frac{\partial(\rho u)}{\partial x}=0 \\
0=-\frac{\partial p}{\partial y}+\left(\frac{\mu}{3}+\mu^{\prime}\right) \frac{\partial^{2} u}{\partial x \partial y} \\
\rho u \frac{\partial u}{\partial x}=-\frac{\partial p}{\partial x}+\left(\frac{4 \mu}{3}+\mu^{\prime}\right) \frac{\partial^{2} u}{\partial x^{2}}+\mu \frac{\partial^{2} u}{\partial y^{2}} \\
\rho c_{p} u \frac{\partial T}{\partial x}-\alpha_{p} T u \frac{\partial p}{\partial x}=\kappa\left(\frac{\partial^{2} T}{\partial x^{2}}+\frac{\partial^{2} T}{\partial y^{2}}\right)+\Phi+\mu^{\prime}\left(\frac{\partial u}{\partial x}\right)^{2}
\end{gathered}
$$

where the governing variables of $\rho, T, p$ and $u$ are density, static temperature, static pressure, and $x$ coordinate velocity of fluid, respectively, $\mu$ ' the bulk viscosity, $\alpha_{p}$ the thermal expansion coefficient, and $\Phi$ the dissipation related to shear viscosity

$$
\alpha_{p}=-\frac{1}{\rho}\left(\frac{\partial \rho}{\partial T}\right)_{p}, \quad \Phi=\mu\left(\frac{\partial u}{\partial y}\right)^{2}+\frac{4}{3} \mu\left(\frac{\partial u}{\partial x}\right)^{2} \quad \text { if }(v \equiv 0)
$$

And two assumptions are also made:

(e) The fluid is ideal gas, as a result, $\alpha_{p} T=1$.

(f) The gas pressure varies only along the Poiseuille flow direction, i.e. $p=p(x)$. The safety of this assumption for mini-/microchannel flow was validated even in the critical conditions of very compressible fluids and high Mach number [12,13,14]. Eq. (2) is therefore omitted to avoid its incompatibility with $p=p(x)$, and the safety of this treatment was also validated by Vandenberg et al. $[12,13]$. Note that we do not ask for the linear pressure profile as that used in $[12,13]$, and we will prove the assumption of linear pressure profile hereafter.

Define the following dimensionless quantities

$$
R e=\frac{\mu}{\rho_{\infty} u_{\infty} d_{e}}, \quad \gamma_{d D}=\frac{d_{e}}{D}, \quad \gamma_{L D}=\frac{L}{D}, \operatorname{Pr}=\frac{\mu c_{p}}{\kappa}, P e=\operatorname{RePr}, \quad E c=\frac{u_{\infty}^{2}}{c_{p}(\Delta T)_{0}}
$$

where $R e$ is the Reynolds number, $d_{e}$ the hydraulic diameter and $d_{e}=2 D$ for an infinite parallel- 
plate channel, $P r$ the Prandtl number, $P e$ the Peclet number, $E c$ is the Eckert number in which the reference temperature rise $(\Delta T)_{0}$ is defined as

$$
(\Delta T)_{0}= \begin{cases}\frac{1}{L} \int_{0}^{L} T_{\mathrm{w}}(x) d x-T_{\infty} & (\mathrm{WT}) \\ -\frac{2}{\rho_{\infty} u_{\infty} c_{p} D} \int_{0}^{L} q(x) d x & (\mathrm{WHF})\end{cases}
$$

Here, 'WT' and 'WHF' represent the two cases of boundary conditions as shown in Fig. 1, i.e. prescribed wall temperature (WT) or prescribed wall heat flux (WHF), in which $T_{\mathrm{w}}(x)$ is the wall temperature in the WT case, $q(x)$ the wall heat flux in the WHF case.. When $T_{\mathrm{w}}(x)=T_{\infty}$ or $q(x)=0$, $(\Delta T)_{0}$ can be arbitrarily taken as a non-zero value, like $(\Delta T)_{0}=T_{\infty}$, to avoid the singularity in mathematics, but in this case the Eckert number does not denote the ratio of twice of the adiabatic temperature rise to the actual temperature rise [15].

Further define the dimensionless variables

$$
\chi=\frac{x}{L}, \quad \xi=\frac{y}{D}, \bar{\rho}=\frac{\rho}{\rho_{\infty}}, \bar{u}=\frac{u}{u_{\infty}}, \bar{p}=\frac{p-p_{\infty}}{\rho_{\infty} u_{\infty}^{2}}, \quad \theta=\frac{T-T_{\infty}}{(\Delta T)_{0}}
$$

where $\rho_{\infty}, p_{\infty}, T_{\infty}$ and $u_{\infty}$ are the gas density, pressure, temperature and mean velocity at the entrance of the channel, respectively, we then get the dimensionless governing equations for continuity and the gas temperature

$$
\begin{gathered}
\frac{\partial(\bar{\rho} \bar{u})}{\partial \chi}=0 \\
\bar{\rho} \bar{u} \frac{\partial \theta}{\partial \chi}=\frac{\gamma_{L D} \gamma_{d D}}{P e}\left(\frac{\partial^{2} \theta}{\partial \xi^{2}}+\frac{1}{\gamma_{L D}^{2}} \frac{\partial^{2} \theta}{\partial \chi^{2}}\right)+E c \bar{u} \frac{d \bar{p}}{d \chi} \\
+E c \frac{\gamma_{L D} \gamma_{d D}}{\operatorname{Re}}\left[\left(\frac{\partial \bar{u}}{\partial \xi}\right)^{2}+\frac{1}{\gamma_{L D}^{2}}\left(\frac{4}{3}+\frac{\mu}{\mu}\right)\left(\frac{\partial \bar{u}}{\partial \chi}\right)^{2}\right]
\end{gathered}
$$

For geometry of a minichannel, the depth of the channel $(D)$ is much smaller than its length $(L)$, i.e. $1 / \gamma_{L D}=D / L$ is normally in order of $10^{-3} \sim 10^{-2}$. Therefore, the items of $\partial^{2} / \partial x^{2}$ and $(\partial u / \partial x)^{2}$ can be omitted and the system holds the framework as the classical boundary-layer equations [15], based on the scale analysis, i.e.

$$
\begin{gathered}
\bar{\rho} \bar{u} \frac{\partial \bar{u}}{\partial \chi}=-\frac{d \bar{p}}{d \chi}+\frac{\gamma_{L D} \gamma_{d D}}{\operatorname{Re}} \frac{\partial^{2} \bar{u}}{\partial \xi^{2}} \\
\bar{\rho} \bar{u} \frac{\partial \theta}{\partial \chi}=\frac{\gamma_{L D} \gamma_{d D}}{P e} \frac{\partial^{2} \theta}{\partial \xi^{2}}+E c \bar{u} \frac{d \bar{p}}{d \chi}+E c \frac{\gamma_{L D} \gamma_{d D}}{R e}\left(\frac{\partial \bar{u}}{\partial \xi}\right)^{2}
\end{gathered}
$$

Correspondingly, the original elliptic system becomes a parabolic one, as a result, only the boundary condition at the channel inlet $(x=0)$ is need in the $x$ direction. We will mainly solve the profiles based on the simplified set of equations, cf. Eqs. (11) and (12), and make improvements with some minor corrections accounting for the axial thermal conduction in Eq. (10).

The corresponding boundary conditions are as follows accounting for no-slip flow at the wall and 
symmetry at the centerline, the dimensionless boundary conditions are

$$
\begin{aligned}
& \theta(0, \xi)=0, \quad \frac{\partial \theta(\chi, 0)}{\partial \xi}=0 \\
& \left\{\begin{array}{l}
\theta\left(\chi, \frac{1}{2}\right)=\theta_{\mathrm{w}}(\chi)=\left[T_{\mathrm{w}}(\chi)-T_{\infty}\right] / \Delta T_{0} \\
\frac{\partial \theta\left(\chi, \frac{1}{2}\right)}{\partial \xi}=-\frac{D}{\kappa(\Delta T)_{0}} q(x)
\end{array}\right.
\end{aligned}
$$

\section{Analytical approximation}

To get analytical solutions, we further assume:

(g) The mass flow is locally fully developed, i.e. the profile of the mass flux is the same as the one obtained for a fully developed incompressible flow. Vandenberg et al. $[12,13]$ used and validated such an assumption in isothermal compressible capillary flow, Guo and $\mathrm{Wu}$ [16] also presented that the locally fully developed approximation is valid if the flow Mach number is moderate.

In mathematical form, considering the continuity equation (cf. Eq. (9)) and the parabolic velocity profile of an incompressible parallel flow in a straight channel, that is

$$
\bar{\rho}(\chi, \xi) \bar{u}(\chi, \xi)=W(\xi)=\frac{3}{2} E(\xi), \quad E(\xi)=1-4 \xi^{2}
$$

Eq. (12) then becomes

$$
\frac{3}{2}\left(1-4 \xi^{2}\right) \frac{\partial \theta}{\partial \chi}=\frac{\gamma_{L D} \gamma_{d D}}{P e} \frac{\partial^{2} \theta}{\partial \xi^{2}}+E c\left[\bar{u} \frac{d \bar{p}}{d \chi}+\frac{\gamma_{L D} \gamma_{d D}}{\operatorname{Re}}\left(\frac{\partial \bar{u}}{\partial \xi}\right)^{2}\right]
$$

The two items in the right hand side of the above equation denote heat conduction and dissipation heat, therefore in physics, the excess temperature is contributed by two effects: the effect of gas/wall heat exchange without dissipation, and the effect of frictional heat with respect to adiabatic wall [15]. In mathematics, we take a linear superposition of zero-order and first-order parts with respect to $E c$, which is normally small enough to be as the perturbation variable (e.g. $u_{\infty}=10 \mathrm{~m} \mathrm{~s}^{-1}, c_{p}=$ $1006 \mathrm{~J} \mathrm{~kg}^{-1} \mathrm{~K}^{-1}$ for air, $(\Delta T)_{0}=10 \mathrm{~K}, E c=0.01$ )

$$
\theta=\theta_{0}+E c \theta_{1}
$$

The Eckert number can be further related to the Mach number of the infinity flow $\left(M a_{\infty}\right)$ with respect to the infinity velocity $\left(u_{\infty}\right)$ and the sound velocity at $T_{\infty}$, i.e. $E c=(\gamma-1)\left(M a_{\infty}\right)^{2} T_{\infty} /(\Delta T)_{0}$, in which $\gamma$ is the specific heat ratio, $\gamma=1.4$ for diatomic gas molecules. At the low and moderate Mach number, or even at the high Mach number but meanwhile the high temperature rise $(\Delta T)_{0}, E c$ is small and the gas/solid heat exchange overwhelms the dissipation heat to dominate the gas temperature rise.

\subsection{Zero-order solutions}

\subsubsection{Prescribed wall-temperature (WT) case}

The zero-order system is 


$$
\left\{\begin{array}{l}
\frac{3}{2}\left(1-4 \xi^{2}\right) \frac{\partial \theta_{0}}{\partial \chi}=\frac{\gamma_{L D} \gamma_{d D}}{P e} \frac{\partial^{2} \theta_{0}}{\partial \xi^{2}} \\
\mathrm{BC}: \theta_{0}(0, \xi)=0, \quad \frac{\partial \theta_{0}(\chi, 0)}{\partial \xi}=0, \quad \theta_{0}\left(\chi, \frac{1}{2}\right)=\theta_{\mathrm{w}}(\chi)
\end{array}\right.
$$

Defining

$$
\theta_{0}(\chi, \xi)=\theta_{\mathrm{w}}(\chi)+\omega(\chi, \xi)
$$

it yields a system with homogeneous boundary conditions

$$
\left\{\begin{array}{l}
\frac{3}{2}\left(1-4 \xi^{2}\right) \frac{\partial \omega}{\partial \chi}=\frac{\gamma_{L D} \gamma_{d D}}{P e} \frac{\partial^{2} \omega}{\partial \xi^{2}}-\frac{3}{2} \frac{d \theta_{\mathrm{w}}}{d \chi}\left(1-4 \xi^{2}\right) \\
\mathrm{BC}: \omega(0, \xi)=-\theta_{\mathrm{w}}(0), \frac{\partial \omega(\chi, 0)}{\partial \xi}=0, \omega\left(\chi, \frac{1}{2}\right)=0
\end{array}\right.
$$

With respect to the corresponding homogeneous equation of the above equation, using separationof-variables technique, we construct

$$
\omega(\chi, \xi)=\sum_{n=1}^{\infty} \omega_{n}(\chi) \psi_{n}(\xi)
$$

which satisfies

$$
\frac{d^{2} \psi_{n}}{d \xi^{2}}=-\lambda_{n}^{2}\left(1-4 \xi^{2}\right) \psi_{n}
$$

Eq. (21) gives an exact solution in the form of Whittaker function, a kind of confluent hypergeometric function [17],

$$
\psi_{n}(\xi)=\frac{1}{\sqrt{\xi}}\left[C_{1 n} M_{\frac{\lambda_{n}}{8}, \frac{1}{4}}\left(2 \lambda_{n} \xi^{2}\right)+C_{2 n} M_{\frac{\lambda_{n}}{8},-\frac{1}{4}}\left(2 \lambda_{n} \xi^{2}\right)\right]
$$

The standard solution of Whittaker's equation is generally expressed as a linear combination of the two linearly independent solutions $M_{\kappa, \mu}(z)$ and $W_{\kappa, \mu}(z)$, seen also Appendix. Some studies used such mathematics for incompressible thermal flow accounting for the parabolic profile of velocity $[18,19]$. Alternatively, we instead use a linear combination of $M_{\kappa, \mu}(z)$ and $M_{\kappa,-\mu}(z)$, as they are linear independent with respect to $\mu=1 / 4$, and it gives us a simpler way to determine the eigenvalues and correspondingly more compact form of eigenfunctions.

Considering the limiting form of $M_{\kappa, \mu}(z)$, cf. Eq. (A3), there is

$$
\psi_{n}(\xi \rightarrow 0)=C_{1 n}\left(2 \lambda_{n}\right)^{\frac{3}{4}} \xi+C_{2 n}\left(2 \lambda_{n}\right)^{\frac{1}{4}}
$$

As a result, the boundary conditions give us $C_{1 n}=0$ and

$$
M_{\frac{\lambda_{n}}{8},-\frac{1}{4}}\left(\frac{\lambda_{n}}{2}\right)=0
$$

which is used to determine the eigenvalues $\lambda_{n}$, and correspondingly the eigenfunctions $\left\{Y_{n}\right\}$ 


$$
Y_{n}(\xi)=\frac{1}{\sqrt{\xi}} M_{\frac{\lambda_{n}}{8},-\frac{1}{4}}\left(2 \lambda_{n} \xi^{2}\right), \quad Y_{n}(\xi \rightarrow 0)=\left(2 \lambda_{n}\right)^{\frac{1}{4}}
$$

Table 1 lists the values of the first fifteen non-zero $\lambda_{n}(n=1 \sim 15)$. It presents a rapidly equallyspaced feature between two neighbor eigenvalues $\left(\lambda_{n}-\lambda_{n-1} \approx 8\right)$, corresponding to the periodically oscillation of $M_{x / 8,-1 / 4}(x / 2)$, as shown in Fig. 2.

Table 1 The first 15 eigenvalues and coefficients for WT case

\begin{tabular}{cccc}
\hline$n$ & $\lambda_{n}$ & $\lambda_{n}-\lambda_{n-1}$ & $a_{1 n}$ \\
\hline 1 & 3.363190644477972 & - & 0.745652186583203 \\
2 & 11.339714691790149 & 7.976524047312177 & -0.137087067881769 \\
3 & 19.336484925020809 & 7.996770233230659 & 0.064491968114589 \\
4 & 27.335322885215088 & 7.998837960194280 & -0.039510622244401 \\
5 & 35.334747130698553 & 7.999424245483464 & 0.027469857769128 \\
6 & 43.334410648649573 & 7.999663517951021 & -0.020574322246476 \\
7 & 51.334192972667623 & 7.999782324018050 & 0.016184902033439 \\
8 & 59.334042089371408 & 7.999849116703786 & -0.013182960291618 \\
9 & 67.333932137332994 & 7.999890047961586 & 0.011020446519237 \\
10 & 75.333848912529149 & 7.999916775196155 & -0.009400044885553 \\
11 & 83.3337840124531 & 7.999935099923960 & 0.008147738226243 \\
12 & 91.3337321717271 & 7.999948159274027 & -0.007155497289913 \\
13 & 99.3336899352377 & 7.999957763510523 & 0.006353025717266 \\
14 & 107.3336549485964 & 7.999965013358704 & -0.005692784230661 \\
15 & 115.3336255561839 & 7.999970607587585 & 0.005141573998427 \\
\hline & & &
\end{tabular}

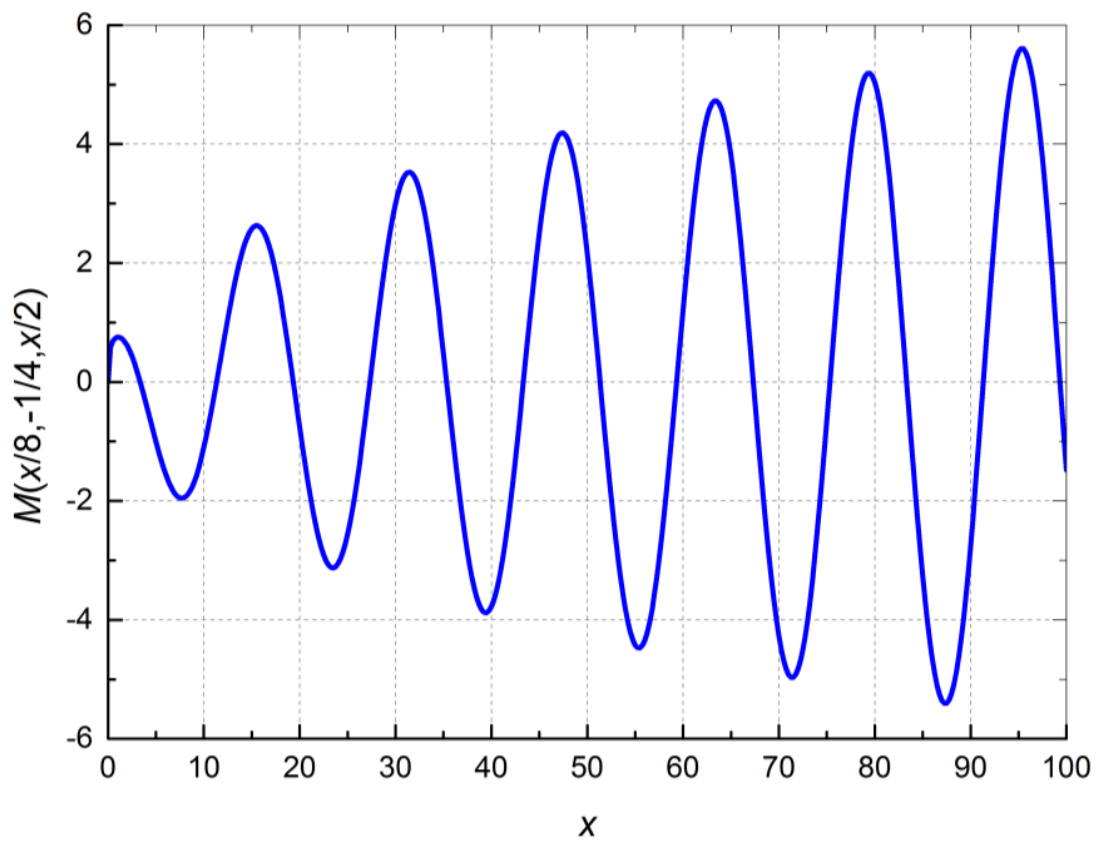

Fig. 2 Zeros and periodically oscillation of Whittaker function $M_{x / 8,-1 / 4}(x / 2)$ 
Expanding the inhomogeneous item (last term) of Eq. (19) with the basis of the eigenfunctions, i.e. $f(\chi, \xi)=\sum f_{n}(\chi) Y_{n}(\xi)$, it yields

$$
\frac{d \omega_{n}}{d \chi}=-b \lambda_{n}^{2} \omega_{n}+f_{n} \quad\left(b=\frac{2 \gamma_{L D} \gamma_{d D}}{3 P e}\right)
$$

of which the solution is

$$
\omega_{n}(\chi)=C_{n} e^{-b_{n} \chi}+e^{-b_{n} \chi} \int_{0}^{\chi} e^{b_{n} \tau} f_{n}(\tau) d \tau
$$

To get a more accurate prediction of the axial profiles, we determine the value of $b_{n}$ from the characteristic equation corresponding to the second-order differential equation (cf. (10))

$$
-\frac{1}{\gamma_{L D}^{2}} b_{n}^{2}+\frac{P e}{\gamma_{L D} \gamma_{d D}} b_{n}+\frac{2}{3} \lambda_{n}^{2}=0
$$

So it yields

$$
b_{n}=\frac{\gamma_{L D}}{2}\left(\sqrt{\left(\frac{P e}{\gamma_{d D}}\right)^{2}+\frac{8}{3} \lambda_{n}^{2}}-\frac{P e}{\gamma_{d D}}\right)
$$

For a limiting case, it reduces to the characteristic constant corresponding to the first-order differential equation (cf. Eq. (26))

$$
b_{n}=b \lambda_{n}^{2} \quad\left(\text { when } \gamma_{L D} \rightarrow \infty\right)
$$

According to Sturm-Liouville theory, the coefficients $f_{n}(\chi)$ and $C_{n}$ are determined by weighted integral of the inhomogeneous item of Eq. (19) and the boundary condition at the inlet $(x=0)$, respectively, using the orthogonality of the eigenfunctions

$$
f_{n}(\chi)=-a_{1 n} \frac{d \theta_{\mathrm{w}}}{d \chi}, \quad C_{n}=-\theta_{\mathrm{w}}(0) a_{1 n}
$$

where

$$
a_{1 n}=\frac{1}{\left\|Y_{n}\right\|^{2}} \int_{0}^{\frac{1}{2}}\left(1-4 \xi^{2}\right) Y_{n}(\xi) d \xi, \quad\left\|Y_{n}\right\|^{2}=\int_{0}^{\frac{1}{2}}\left(1-4 \xi^{2}\right) Y_{n}^{2}(\xi) d \xi
$$

Therefore, the zero-order dimensionless total temperature for WT case is obtained by

$$
\theta_{0}(\chi, \xi)=\theta_{w}\left(1-\sum_{n=1}^{N} a_{1 n} Y_{n}\right)+\sum_{n=1}^{N} \varphi_{n} Y_{n}, \varphi_{n}(\chi)=a_{1 n} b_{n} e^{-b_{n} \chi} \int_{0}^{\chi} e^{b_{n} \tau} \theta_{\mathrm{w}}(\tau) d \tau
$$

in which the first item vanishes as $\sum a_{1 n} Y_{n}(\xi)=1$ when $N \rightarrow \infty$, anyway, it is still kept accounting for the truncation error when limited items (i.e. $N<\infty$ ) are taken for numerical calculation.

Table 1 also lists the values of the first 15 coefficients, $a_{1 n}$, which show a feature of positive and negative alternation and a gradual decrease in magnitude.

The average dimensionless temperature weighted by the mass flow rate is

$$
\theta_{\mathrm{m}}(\chi)=2 \int_{0}^{\frac{1}{2}} W(\xi) \theta_{0}(\chi, \xi) d \xi=\theta_{w}+3 \sum_{n=1}^{N} a_{1 n}\left\|Y_{n}\right\|^{2}\left(\varphi_{n}-a_{1 n} \theta_{w}\right)
$$


The local Nusselt number and the mean Nusselt number can then be obtained by

$$
\begin{gathered}
N u(\chi)=\frac{\gamma_{d D}}{\theta_{\mathrm{w}}(\chi)-\theta_{\mathrm{m}}(\chi)}\left(\frac{\partial \theta_{0}}{\partial \xi}\right)_{\xi=\frac{1}{2}} \\
=-\frac{\gamma_{d D}}{3 \sqrt{2}}\left[\sum_{n=1}^{N} a_{1 n}\left\|Y_{n}\right\|^{2}\left(\varphi_{n}-a_{1 n} \theta_{w}\right)\right]^{-1} \sum_{n=1}^{N}\left(\varphi_{n}-a_{1 n} \theta_{\mathrm{w}}\right)\left(\lambda_{n}+2\right) M_{\frac{\lambda_{n}}{8}+1,-\frac{1}{4}}\left(\frac{\lambda_{n}}{2}\right) \\
N u_{\mathrm{m}}=\int_{0}^{1} N u(\chi) d \chi
\end{gathered}
$$

\subsubsection{Prescribed wall heat flux (WHF) case}

Defining

$$
\theta_{0}(\chi, \xi)=-\frac{D}{\kappa(\Delta T)_{0}} q(\chi) \xi^{2}+\omega(\chi, \xi)
$$

the zero-order system with homogeneous boundary conditions is obtained by

$$
\left\{\begin{array}{l}
\frac{3}{2}\left(1-4 \xi^{2}\right) \frac{\partial \omega}{\partial \chi}=\frac{\gamma_{L D} \gamma_{d D}}{P e} \frac{\partial^{2} \omega}{\partial \xi^{2}}+\frac{D}{\kappa(\Delta T)_{0}}\left[-\frac{2 \gamma_{L D} \gamma_{d D}}{P e} q(\chi)+\frac{3}{2} \frac{d q}{d \chi}\left(1-4 \xi^{2}\right) \xi^{2}\right] \\
\mathrm{BC}: \omega(0, \xi)=\frac{D}{\kappa(\Delta T)_{0}} q(0) \xi^{2}, \quad \frac{\partial \omega(\chi, 0)}{\partial \xi}=0, \quad \frac{\partial \omega\left(\chi, \frac{1}{2}\right)}{\partial \xi}=0
\end{array}\right.
$$

Also using separation-of-variables technique, we construct

$$
\omega(\chi, \xi)=\sum_{n=0}^{\infty} \omega_{n}(\chi) \psi_{n}(\xi)
$$

where Eqs. (21) (23) still hold for $\psi_{n}(\xi)$ when $n \geq 1$. Note that, different to Eq. (20), the index of $n$ in Eq. (39) starts from $n=0$. This difference is due to both the Newman-type boundary conditions at the two ends $(\xi=0$ and $1 / 2)$ in the WHF case, which allows the existence of a nontrivial directcurrent item corresponding to $n=0$, i.e.

$$
Y_{0}(\xi)=1, \quad Y_{n}(\xi)=\frac{1}{\sqrt{\xi}} M_{\frac{\lambda_{n}}{8}, \frac{1}{4}}\left(2 \lambda_{n} \xi^{2}\right) \quad(n \geq 1)
$$

The eigenvalues are determined by the boundary condition, $d Y_{n} / d \xi \mid \xi=1 / 2=0$. According to Eq. (A4), there are

$$
\lambda_{0}=0, \quad\left(\frac{\lambda_{n}}{4}+\frac{1}{2}\right) M_{\frac{\lambda_{n}}{8}+1,-\frac{1}{4}}\left(\frac{\lambda_{n}}{2}\right)+\left(\frac{\lambda_{n}}{4}-\frac{1}{2}\right) M_{\frac{\lambda_{n}}{8},-\frac{1}{4}}\left(\frac{\lambda_{n}}{2}\right)=0 \quad(n \geq 1)
$$

The function $\omega_{n}(\chi)$ holds the same framework as Eqs. (26) and (27). According to Sturm-Liouville theory, the coefficients $f_{n}(\chi)$ and $C_{n}$ are determined by the weighted orthogonal eigenfunctions

$$
f_{n}(\chi)=\frac{D}{\kappa(\Delta T)_{0}}\left[-2 b a_{2 n} q(\chi)+a_{3 n} \frac{d q}{d \chi}\right]
$$




$$
C_{n}=\frac{D q(0)}{\kappa(\Delta T)_{0}} a_{3 n}
$$

where

$$
a_{2 n}=\frac{1}{\left\|Y_{n}\right\|^{2}} \int_{0}^{\frac{1}{2}} Y_{n}(\xi) d \xi, \quad a_{3 n}=\frac{1}{\left\|Y_{n}\right\|^{2}} \int_{0}^{\frac{1}{2}} \xi^{2}\left(1-4 \xi^{2}\right) Y_{n}(\xi) d \xi
$$

Table 2 lists the first 16 eigenvalues and coefficients $a_{2 n}$ and $a_{3 n}$.

Table 2 The first 16 eigenvalues and coefficients for WHF case

\begin{tabular}{ccccc}
\hline$n$ & $\lambda_{n}$ & $\lambda_{n}-\lambda_{n-1}$ & $a_{2 n}$ & $a_{3 n}$ \\
\hline 0 & 0 & - & 1.5 & 0.05 \\
1 & 8.574449891262043 & 8.574449891262043 & -0.415571171308799 & -0.031699493520337 \\
2 & 16.607448955054519 & 8.032999063792476 & 0.259767859813622 & 0.008889805049549 \\
3 & 24.621212125443339 & 8.013763170388820 & -0.194925373536150 & -0.004085635816366 \\
4 & 32.629043392171262 & 8.007831266727923 & 0.158440349195970 & 0.002327688456663 \\
5 & 40.634194492735453 & 8.005151100564191 & -0.134716049103952 & -0.001496984555122 \\
6 & 48.637883309996965 & 8.003688817261512 & 0.117905609077432 & 0.001040849607436 \\
7 & 56.640677408765349 & 8.002794098768383 & -0.105294085812198 & -0.000764220478390 \\
8 & 64.642879897453426 & 8.002202488688077 & 0.095438852691162 & 0.000584142193557 \\
9 & 72.644668596367097 & 8.001788698913671 & -0.087498182354645 & -0.000460521842797 \\
10 & 80.646155277311152 & 8.001486680944055 & 0.080945877577779 & 0.000372077359867 \\
11 & 88.647414016896690 & 8.001258739585538 & -0.075435054203879 & -0.000306669699167 \\
12 & 96.648496014183735 & 8.001081997287045 & 0.070727183475642 & 0.0002569688853360 \\
13 & 104.6494378846336 & 8.000941870449893 & -0.066652512961491 & -0.000218338785437 \\
14 & 112.6502665659481 & 8.000828681314474 & 0.063086739382730 & 0.000187732406313 \\
15 & 120.6510023546735 & 8.000735788725436 & -0.059936621273379 & -0.000163080971660 \\
\hline
\end{tabular}

The final solution of zero-order dimensionless total temperature for WHT case is

$$
\begin{aligned}
\theta_{0}(\chi, \xi) & =-\frac{D}{\kappa(\Delta T)_{0}} q(\chi)\left(\xi^{2}-\sum_{n=0}^{N} a_{3 n} Y_{n}\right)+\sum_{n=0}^{N} \varphi_{n}(\chi) Y_{n}(\xi), \\
\varphi_{n}(\chi) & =-\frac{D}{\kappa(\Delta T)_{0}}\left[\left(2 b a_{2 n}+a_{3 n} b_{n}\right) e^{-b_{n} \chi} \int_{0}^{\chi} e^{b_{n} \tau} q(\tau) d \tau\right]
\end{aligned}
$$

in which the first item vanishes as $\sum a_{3 n} Y_{n}(\xi)=\xi^{2}$ when $N \rightarrow \infty$, anyway, it is still kept accounting for the truncation error as limited items should be taken for a practical numerical calculation.

The average dimensionless temperature, local Nusselt number and the mean Nusselt number can then be obtained by

$$
\begin{gathered}
\theta_{\mathrm{m}}(\chi)=2 \int_{0}^{\frac{1}{2}} W(\xi) \theta_{0}(\chi, \xi) d \xi=-\frac{3 D b}{\kappa(\Delta T)_{0}} \int_{0}^{\chi} q(\tau) d \tau \\
N u(\chi)=-\frac{q(\chi) d_{e}}{\kappa(\Delta T)_{0}\left[\theta_{0}\left(\chi, \frac{1}{2}\right)-\theta_{\mathrm{m}}(\chi)\right]}
\end{gathered}
$$




$$
N u_{\mathrm{m}}=\int_{0}^{1} N u(\chi) d \chi
$$

\subsection{Profiles of pressure, density and velocity}

Going back to the dimensional momentum equation, it is

$$
\rho u \frac{\partial u}{\partial \chi}=-\frac{d p}{d \chi}+\frac{\rho_{\infty} u_{\infty} \gamma_{L D} \gamma_{d D}}{\operatorname{Re}} \frac{\partial^{2} u}{\partial \xi^{2}}
$$

Considering the equation of state of ideal gas and Eq. (14), the gas velocity is related to the local temperature as

$$
\left.\begin{array}{l}
\rho R_{g} T=p(\chi) \\
\rho u=\rho_{\infty} u_{\infty} W(\xi)
\end{array}\right\} \Rightarrow u=\frac{\rho_{\infty} u_{\infty} R_{g} W(\xi)}{p(\chi)} T(\chi, \xi)
$$

where $R_{\mathrm{g}}$ is the gas constant of the fluid, $R_{\mathrm{g}}=287 \mathrm{~J} \mathrm{~kg}^{-1} \mathrm{~K}^{-1}$ for air.

Substituting Eq. (50), Eq. (49) becomes

$$
\left(\frac{1}{\rho_{\infty}^{2} u_{\infty}^{2} R_{g}}-\frac{W^{2} T}{p^{2}}\right) p \frac{d p}{d \chi}=-W^{2} \frac{\partial T}{\partial \chi}+\frac{\gamma_{L D} \gamma_{d D}}{R e}\left(\frac{d^{2} W}{d \xi^{2}} T+2 \frac{d W}{d \xi} \frac{\partial T}{\partial \xi}+W \frac{\partial^{2} T}{\partial \xi^{2}}\right)
$$

Considering

$$
\frac{T}{p^{2}} \approx \frac{1}{p \rho_{\mathrm{avg}} R_{g}}=\frac{1}{p \rho_{\infty} \beta_{\rho} R_{g}}, \quad \beta_{\rho}=1-\frac{1}{2 T_{\infty}}(\Delta T)_{0}+\frac{\rho_{\infty}}{2 T_{\infty}^{2}}\left[(\Delta T)_{0}\right]^{2}
$$

Further, taking an approximation of the gas temperature,

$$
T(\chi, \xi)=T_{\infty}+(\Delta T)_{0} \theta \approx T_{\infty}+(\Delta T)_{0} \theta_{0}
$$

substituting it into Eq. (51) and integrating in the $\xi$ direction with a weight of function $W(\xi)$, we got

$$
\begin{array}{r}
\frac{1}{2 \rho_{\infty}^{2} u_{\infty}^{2} R_{g}} \frac{d}{d \chi}\left[\left(p-\frac{54}{35} \frac{\rho_{\infty} u_{\infty}^{2}}{\beta_{\rho}}\right)^{2}\right]=-\frac{12 \gamma_{L D} \gamma_{d D} T_{\infty}}{\operatorname{Re}}+F(\chi) \\
+(\Delta T)_{0} \sum_{n}\left(-a_{4 n} \frac{d \varphi_{n}}{d \chi}+\frac{\gamma_{L D} \gamma_{d D}}{\operatorname{Re}} a_{5 n} \varphi_{n}\right)
\end{array}
$$

where

$$
\begin{gathered}
a_{4 n}=2 \int_{0}^{1 / 2} W^{3} Y_{n}(\xi) d \xi=\frac{27}{4} \int_{0}^{1 / 2}\left(1-4 \xi^{2}\right)^{3} Y_{n}(\xi) d \xi \\
a_{5 n}=2 \int_{0}^{1 / 2} W \frac{d^{2} W}{d \xi^{2}} Y_{n}(\xi) d \xi=-36 \int_{0}^{1 / 2}\left(1-4 \xi^{2}\right) Y_{n}(\xi) d \xi
\end{gathered}
$$

and

$$
F(\chi)=(\Delta T)_{0}\left[\left(-\frac{54}{35}+\sum_{n=1}^{N} a_{1 n} a_{4 n}\right) \frac{d \theta_{w}}{d \chi}-\frac{\gamma_{L D} \gamma_{d D}}{\operatorname{Re}}\left(12+\sum_{n=1}^{N} a_{1 n} a_{5 n}\right) \theta_{w}(\chi)\right]
$$

for the WT case, or 


$$
F(\chi)=\frac{D}{\kappa}\left(\frac{3}{70}-\sum_{n=0}^{N} a_{3 n} a_{4 n}\right) \frac{d q}{d \chi}
$$

for the WHF case, in which $a_{5 n}=0(n>1)$ has been used accounting for the orthogonality between $Y_{0}(\xi)=1$ and $Y_{n}(\xi)(n>1)$. The function $F(\chi)$ is denoted as the truncation error function, as $\sum a_{1 n} a_{4 n}$ $=54 / 35$ and $\sum a_{1 n} a_{5 n}=-12$ in the WT case, and $\sum a_{3 n} a_{4 n}=3 / 70$ in the WHF case, when $N \rightarrow \infty$.

Eq. (54) principally gives us a nonlinear profile of the gas pressure and correspondingly a linear profile in the cases of moderate temperature rises.

\subsection{First-order total temperature}

The first-order system for the total temperature is

$$
\left\{\begin{aligned}
\frac{3}{2}\left(1-4 \xi^{2}\right) \frac{\partial \theta_{1}}{\partial \chi} & =\frac{\gamma_{L D} \gamma_{d D}}{P e} \frac{\partial^{2} \theta_{1}}{\partial \xi^{2}}+\bar{u} \frac{d \bar{p}}{d \chi}+\frac{\gamma_{L D} \gamma_{d D}}{\operatorname{Re}}\left(\frac{\partial \bar{u}}{\partial \xi}\right)^{2} \\
\mathrm{BC}: \theta_{1}(0, \xi) & =0, \quad \frac{\partial \theta_{1}(\chi, 0)}{\partial \xi}=0 \\
\theta_{1}\left(\chi, \frac{1}{2}\right) & =0(\mathrm{WT}) \text { or } \frac{\partial \theta_{1}\left(\chi, \frac{1}{2}\right)}{\partial \xi}=0(\mathrm{WHF})
\end{aligned}\right.
$$

The system with the homogeneous boundary conditions still holds the Whittaker's feature as the same as the zero-order system, i.e. Eqs. (24) and (25) or and Eqs. (40) and (41) hold for the eigenvalues and eigenfunctions for the WT and WHF case, respectively.

Substituting Eqs. (50) in (59), it yields

$$
\frac{3}{2}\left(1-4 \xi^{2}\right) \frac{\partial \theta_{1}}{\partial \chi}=\frac{\gamma_{L D} \gamma_{d D}}{P e} \frac{\partial^{2} \theta_{1}}{\partial \xi^{2}}+\frac{\rho_{\infty} R_{g} W T}{p} \frac{d \bar{p}}{d \chi}+\frac{\gamma_{L D} \gamma_{d D}}{R e} \frac{\rho_{\infty}^{2} R_{g}^{2}}{p^{2}}\left(-12 \xi T+W \frac{\partial T}{\partial \xi}\right)^{2}
$$

Using the temperature profile (cf. Eq. (53)) and the pressure profile (cf. Eq. (54)), expanding all the items in Eq. (59) in the basis of the eigenfunctions, following the Sturm-Liouville theory for weighted orthogonality of the eigenfunctions, we can in principle solve the first-order solution. Considering the tedious mathematical deviation, we instead present a simplified algorithm introducing below.

\subsection{Simplified algorithm}

In the preceding context, the fully two-dimensional profile of the gas density, i.e. $\rho=\rho(x, y)$ was considered for strict mathematical deviations. As a matter of fact, due to the large length-depth ratio of a minichannel, the gas density in the $y$ direction develops rapidly approaching to be homogenous except for the region very close to the channel inlet. Therefore, in this section, the gas density is assumed to be just function of $x$ coordinate, i.e. $\rho=\rho_{m}(x)$. Although it is incompatible with the equation of the state, i.e. $\rho=p(x) / R_{\mathrm{g}} T(x, y)$ for ideal gases, the weakness of this assumption is considered to be insignificant accounting for the thermal expansion coefficient, $\alpha_{p}=1 / T$, which means a relative variation of the gas density in $y$ direction in order of $10^{-3}$.

\subsubsection{Profiles of velocity and density}

Still with the parabolic velocity profile, i.e. $u=u_{m}(x) E(y)$ where $u_{m}(x)$ denotes the centerline (maximum in the $y$ direction) velocity, the continuity equation leads to 


$$
\bar{\rho}_{m}(\chi) \bar{u}(\chi, \xi)=\bar{\rho}_{m}(\chi) \bar{u}_{m}(\chi) E(\xi)=W(\xi) \quad \Rightarrow \quad \bar{\rho}_{m} \bar{u}_{m}=\frac{3}{2}
$$

The zero-order temperature solution in the preceding mathematics is not affected. Revisiting the momentum equation, multiplying with $\rho(x)$ at the both sides of Eq. (11), we got

$$
-\left(\bar{\rho}_{m} \bar{u}_{m}\right)^{2} E^{2} \frac{\partial \ln \bar{\rho}_{m}}{\partial \chi}=-\bar{\rho}_{m} \frac{d \bar{p}}{d \chi}+\frac{\gamma_{L D} \gamma_{d D}}{\operatorname{Re}} \bar{\rho}_{m} \bar{u}_{m} \frac{\partial^{2} E}{\partial \xi^{2}}
$$

Via integrating the above equation in the $y$ direction weighted by the mass flow rate, it yields

$$
\frac{d \bar{u}_{m}}{d \chi}=-a_{0} \bar{u}_{m}-\frac{35}{36} \frac{d \bar{p}}{d \chi} \quad\left(a_{0}=\frac{70 \gamma_{L D} \gamma_{d D}}{9 R e}\right)
$$

Accounting for the pressure profile, which is generally a combination of power functions and exponent functions (cf. Eq. (54) and see also the Section 4), i.e.

$$
\frac{d \bar{p}}{d \chi}=\sum_{j=0}^{K} \beta_{j} \chi^{j}+\sum_{j=0}^{K_{2}} \alpha_{m} e^{-\hbar}
$$

we can get the velocity and density profiles as

$$
\begin{gathered}
\bar{u}_{m}(\chi)=\frac{3}{2} e^{-a_{0} \chi}-\frac{35}{36} \sum_{j=0}^{K} \beta_{j}\left[\frac{(-1)^{j+1} j !}{\left(a_{0}\right)^{j+1}} e^{-a_{0} \chi}+\sum_{k=0}^{j} \frac{(-1)^{k}(j)_{k}}{\left(a_{0}\right)^{k+1}} \chi^{j-k}\right] \\
-\frac{35}{36} \sum_{j=0}^{K_{2}} \frac{\alpha_{j}}{a_{0}-\hbar}\left(e^{-\hbar}-e^{-a_{0} \chi}\right) \\
\bar{\rho}_{m}(\chi)=3 / 2 \bar{u}_{m}
\end{gathered}
$$

\subsubsection{First-order total temperature}

When $\rho=\rho_{m}(x)$, Eq. (59) becomes

$$
\frac{3}{2}\left(1-4 \xi^{2}\right) \frac{\partial \theta_{1}}{\partial \chi}=\frac{\gamma_{L D} \gamma_{d D}}{P e} \frac{\partial^{2} \theta_{1}}{\partial \xi^{2}}+\bar{u}_{m}\left(1-4 \xi^{2}\right) \frac{d \bar{p}}{d \chi}+\frac{64 \gamma_{L D} \gamma_{d D}}{\operatorname{Re}} \bar{u}_{m}^{2} \xi^{2}
$$

Following the preceding mathematical framework, the first-order temperature profile is easily obtained by expanding all the items in the basis of the eigenfunctions as

$$
\begin{aligned}
& \theta_{1}=\sum_{n=1, \mathrm{or} 0}^{N_{1}} \varphi_{1 n}(\chi) Y_{n}(\xi) \\
& \varphi_{1 n}(\chi)=e^{-b_{n} \chi} \int_{0}^{\chi} e^{b_{n} \tau}\left(\frac{2}{3} \bar{u}_{m} \frac{d \bar{p}}{d \tau} a_{1 n}+\frac{128 \gamma_{L D} \gamma_{d D}}{3 R e} \bar{u}_{m}^{2} a_{6 n}\right) d \tau
\end{aligned}
$$

where

$$
a_{6 n}=\frac{1}{\left\|Y_{n}\right\|^{2}} \int_{0}^{1 / 2} \xi^{2} Y_{n}(\xi) d \xi
$$

The index $n$ starts from $n=1$ or 0 in the WT and WHF cases, respectively, to $N_{1}$, the number of terms. In the WHF case, $a_{1 n}=0(n>1)$ holds due to the orthogonality between $Y_{0}(\xi)=1$ and $Y_{n}(\xi)$ 
$(n>1)$. Note that again, we take the same symbol sets for the WT and the WHF cases, but the values of the variables are different because of the different eigenvalues $\left\{\lambda_{n}\right\}$ and eigenfunctions $\left\{Y_{n}\right\}$.

The above algorithm can be further simplified. Multiplying Eq. (11) with $\bar{u}$ and summing it with Eq. (12), we got

$$
\bar{\rho} \bar{u} \frac{\partial \theta_{\mathrm{t}}}{\partial \chi}=\frac{\gamma_{L D} \gamma_{d D}}{P e} \frac{\partial^{2} \theta_{\mathrm{t}}}{\partial \xi^{2}}+E c \frac{\gamma_{L D} \gamma_{d D}}{\operatorname{Re}}\left(1-\frac{1}{P r}\right) \frac{\partial}{\partial \xi}\left(\bar{u} \frac{\partial \bar{u}}{\partial \xi}\right)
$$

where $\theta_{\mathrm{t}}$ denotes the dimensionless total (or stagnant) temperature

$$
T_{\mathrm{t}}=T+\frac{u^{2}}{2 c_{p}}, \quad \theta_{\mathrm{t}}=\frac{T_{\mathrm{t}}-T_{\infty}}{(\Delta T)_{0}}=\theta+\frac{1}{2} E c \bar{u}^{2}
$$

With the linear perturbation expansion of $\theta_{\mathrm{t}}$

$$
\theta_{\mathrm{t}}=\theta_{\mathrm{t}, 0}+E c \theta_{\mathrm{t}, 1}, \quad \theta_{\mathrm{t}, 0}=\theta_{0}, \quad \theta_{\mathrm{t}, 1}=\theta_{1}+\frac{1}{2} \bar{u}_{0}^{2}
$$

in which the zero-order velocity $u_{0}=u_{m}(x) E(y)$, the differential system of the first-order total temperature is therefore obtained by

$$
\left\{\begin{aligned}
\frac{3}{2}\left(1-4 \xi^{2}\right) \frac{\partial \theta_{\mathrm{t}, 1}}{\partial \chi} & =\frac{\gamma_{L D} \gamma_{d D}}{P e} \frac{\partial^{2} \theta_{\mathrm{t}, 1}}{\partial \xi^{2}}-\frac{8 \gamma_{L D} \gamma_{d D}}{R e}\left(1-\frac{1}{P r}\right) \bar{u}_{m}^{2}\left(1-12 \xi^{2}\right) \\
\mathrm{BC}: \quad \theta_{\mathrm{t}, 1}(0, \xi) & =\frac{9}{8}\left(1-4 \xi^{2}\right)^{2}, \quad \frac{\partial \theta_{1}(\chi, 0)}{\partial \xi}=0 \\
\theta_{\mathrm{t}, 1}\left(\chi, \frac{1}{2}\right) & =0(\mathrm{WT}) \text { or } \frac{\partial \theta_{\mathrm{t}, 1}\left(\chi, \frac{1}{2}\right)}{\partial \xi}=0(\mathrm{WHF})
\end{aligned}\right.
$$

Compared to Eq. (67), the differential system is simplified without the pressure gradient. By using the same mathematical framework, it yields the corresponding solution as

$$
\begin{aligned}
& \theta_{\mathrm{t}, 1}=\sum_{n=1, \mathrm{or} 0}^{N_{1}} \varphi_{\mathrm{t}, n}(\chi) Y_{n}(\xi) \\
& \varphi_{\mathrm{t}, n}(\chi)=a_{7 n} e^{-b_{n} \chi}-\frac{16 \gamma_{L D} \gamma_{d D}}{3 R e}\left(1-\frac{1}{P r}\right) e^{-b_{n} \chi} \int_{0}^{\chi} a_{8 n} e^{b_{n} \tau} \bar{u}_{m}^{2}(\tau) d \tau
\end{aligned}
$$

where

$$
a_{7 n}=\frac{1}{\left\|Y_{n}\right\|^{2}} \int_{0}^{1 / 2} \frac{9}{8}\left(1-4 \xi^{2}\right)^{3} Y_{n}(\xi) d \xi, \quad a_{8 n}=\frac{1}{\left\|Y_{n}\right\|^{2}} \int_{0}^{1 / 2}\left(1-12 \xi^{2}\right) Y_{n}(\xi) d \xi
$$

The first-order temperature is finally simplified to

$$
\theta_{1}=\theta_{\mathrm{t}, 1}-\frac{1}{2} \bar{u}_{m}^{2}\left(1-4 \xi^{2}\right)^{2}
$$

\section{Applications}

In this section, we apply the preceding mathematical framework to two specific cases, with the prescribed wall temperature or wall heat flux in power-law profile, which are without losing of generality accounting for Taylor-series expansion of continuous rational functions. Then the 
analytical solutions are compared with the exact (numerical) computations, in the cases of constant wall temperature or constant heat flux. In the text below, only the zero-order solutions are included for a compact expression, as the first-order solutions contribute very little at small values of $E c$. Finally, a calculation is employed to validate the algorithms at moderate Reynolds number and Mach number, in which situation the friction heat (i.e. the first-order solution) could dominate the gas temperature profile.

Case 1: the excess temperature of the wall presents a power-law distribution, in this case, similar solutions exist in the classical boundary-layer problems.

$$
T_{\mathrm{w}}(\chi)-T_{\infty}=\sum_{m=0}^{M} c_{m} \chi^{m}, \quad \theta_{\mathrm{w}}(\chi)=\sum_{m=0}^{M} c_{m} \chi^{m} /(\Delta T)_{0}, \quad(\Delta T)_{0}=\sum_{m=0}^{M} \frac{c_{m}}{m+1}
$$

Following Eq. (33) and Eq. (B3),

$$
\begin{aligned}
\theta_{0}(\chi, \xi) & =\frac{1}{(\Delta T)_{0}} \sum_{m=0}^{M} c_{m} \chi^{m}\left(1-\sum_{n=1}^{N} a_{1 n} Y_{n}\right)+\sum_{n=1}^{N} \varphi_{n} Y_{n}(\xi) \\
\varphi_{n}(\chi) & =\frac{a_{1 n}}{(\Delta T)_{0}} \sum_{m=0}^{M} c_{m}\left[\sum_{k=0}^{m} \frac{(-1)^{k}(m)_{k}}{\left(b_{n}\right)^{k}} \chi^{m-k}+\frac{(-1)^{m+1} m !}{\left(b_{n}\right)^{m}} e^{-b_{n} \chi}\right]
\end{aligned}
$$

Using Eqs. (35) and (36), the Nusselt number is

$$
\begin{aligned}
N u(\chi)= & -\frac{\gamma_{d D}}{3 \sqrt{2}}\left[\sum_{n=1}^{N} a_{1 n}\left\|Y_{n}\right\|^{2}\left(\varphi_{n}-\frac{a_{1 n}}{(\Delta T)_{0}} \sum_{m=0}^{M} c_{m} \chi^{m}\right)\right]^{-1} \times \\
& \sum_{n=1}^{N}\left(\varphi_{n}-\frac{a_{1 n}}{(\Delta T)_{0}} \sum_{m=0}^{M} c_{m} \chi^{m}\right)\left(\lambda_{n}+2\right) M_{\frac{\lambda_{n}}{8}+1,-\frac{1}{4}}\left(\frac{\lambda_{n}}{2}\right)
\end{aligned}
$$

Following Eq. (54), the pressure profile is

$$
\begin{array}{r}
\frac{u_{\infty}^{2}}{2 R_{g}} \frac{d}{d \chi}\left[\left(\bar{p}+\frac{p_{\infty}}{\rho_{\infty} u_{\infty}^{2}}-\frac{54}{35 \beta_{\rho}}\right)^{2}\right]=-\frac{12 \gamma_{L D} \gamma_{d D} T_{\infty}}{\operatorname{Re}}+F(\chi) \\
+(\Delta T)_{0} \sum_{n=1}^{N}\left(-a_{4 n} \phi_{n}+\frac{\gamma_{L D} \gamma_{d D}}{\operatorname{Re}} a_{5 n} \varphi_{n}\right)
\end{array}
$$

where

$$
\begin{aligned}
& F(\chi)=\left(-\frac{54}{35}+\sum_{n=1}^{N} a_{1 n} a_{4 n}\right) \sum_{m=1}^{M} m c_{m} \chi^{m-1}-\frac{\gamma_{L D} \gamma_{d D}}{R e}\left(12+\sum_{n=1}^{N} a_{1 n} a_{5 n}\right) \sum_{m=0}^{M} c_{m} \chi^{m} \\
& \phi_{n}(\chi)=\frac{d \varphi_{n}}{d \chi}=\frac{a_{1 n}}{(\Delta T)_{0}} \sum_{m=0}^{M} c_{m}\left[\sum_{k=0}^{m-1} \frac{(-1)^{k}(m)_{k+1}}{\left(b_{n}\right)^{k}} \chi^{m-k-1}+\frac{(-1)^{m} m !}{\left(b_{n}\right)^{m-1}} e^{-b_{n} \chi}\right]
\end{aligned}
$$

Specially, for a constant wall temperature, i.e. $T_{\mathrm{w}}=$ Const and $\theta_{\mathrm{w}}=1(m=0)$, there are

$$
\theta_{0}(\chi, \xi)=1-\sum_{n=1}^{N} a_{1 n} e^{-b_{n} \chi} Y_{n}(\xi)
$$




$$
\begin{gathered}
N u(\chi)=-\frac{\gamma_{d D}}{3 \sqrt{2}}\left(\sum_{n=1}^{N} a_{1 n}^{2}\left\|Y_{n}\right\|^{2} e^{-b_{n} \chi}\right)^{-1} \sum_{n=1}^{N} a_{1 n}\left(\lambda_{n}+2\right) M_{\frac{\lambda_{n}}{8}+1,-\frac{1}{4}}\left(\frac{\lambda_{n}}{2}\right) e^{-b_{n} \chi} \\
\frac{u_{\infty}^{2}}{2 R_{g}} \frac{d}{d \chi}\left[\left(\bar{p}+\frac{p_{\infty}}{\rho_{\infty} u_{\infty}^{2}}-\frac{54}{35 \beta_{\rho}}\right)^{2}\right]=\frac{-12 \gamma_{L D} \gamma_{d D} T_{w}}{\operatorname{Re}} \\
-(\Delta T)_{0} \sum_{n=1}^{\infty} a_{1 n}\left(a_{4 n} b_{n}+\frac{\gamma_{L D} \gamma_{d D}}{R e} a_{5 n}\right) e^{-b_{n} \chi}
\end{gathered}
$$

In the case of the moderate temperature difference, $(\Delta T)_{0}$, the second term in the right hand side of Eq. (86) is normally small, a linear pressure profile can then be obtained

$$
\frac{d \bar{p}}{d \chi}=\frac{-12 \gamma_{L D} \gamma_{d D}}{\operatorname{Re}\left(1-\frac{54}{35 \beta_{\rho}} \gamma M a_{\infty}^{2}\right)}\left[1+\frac{(\Delta T)_{0}}{T_{\infty}}\right]
$$

which can be further simplified at the moderate Mach number as

$$
\frac{d \bar{p}}{d \chi}=\frac{-12 \gamma_{L D} \gamma_{d D}}{\operatorname{Re}}\left[1+\frac{(\Delta T)_{0}}{T_{\infty}}\right]
$$

The velocity and density profile can then be calculated by Eq. (50) and (14). The centerline velocity can be obtained based on the simplified algorithm, cf. Eq. (65)

$$
\bar{u}_{m}(\chi)=\frac{3}{2} e^{-a_{0} \chi}-\frac{35}{36 a_{0}} \beta_{0}\left(1-e^{-a_{0} \chi}\right), \quad \beta_{0}=\frac{-12 \gamma_{L D} \gamma_{d D}}{\operatorname{Re}\left(1-\frac{54}{35 \beta_{\rho}} \gamma M a_{\infty}^{2}\right)}\left[1+\frac{(\Delta T)_{0}}{T_{\infty}}\right]
$$

which can be used to calculate the first-order temperature, cf. Eq. (68).

Figure 3 shows the analytical and exact along-the-channel profiles of the dimensionless gas temperature at the centerline, the average density, centerline velocity, pressure gradient and the local Nusselt number in the constant WT case. The parameters are: $D=10^{-3} \mathrm{~m}, L=0.05 \mathrm{~m}, u_{\infty}=0.5 \mathrm{~m}$ $\mathrm{s}^{-1}, p_{\infty}=10^{5} \mathrm{~Pa}, T_{\infty}=300 \mathrm{~K}, T_{\mathrm{w}}=400 \mathrm{~K},(\Delta T)_{0}=100 \mathrm{~K}$, which correspond to the dimensionless parameters as $\gamma_{L D}=50, \operatorname{Pr}=0.744, \operatorname{Re}=64.9, M a_{\infty}=0.0014, E c=2.48 \times 10^{-6}$ for air. The exact solutions were obtained by using the commercial package, COMSOL [20], and the number of items $N=5$ was taken in default for the analytical computations. The algorithms, including the simplified algorithm, were validated well. As shown in Fig. 3b, the Nusselt number approaches to $N u=7.54$, which agrees very well with the theoretical value for the fully-developed thermal flow in a rectangular duct.

As shown in Fig. 4a, the profiles of the dimensionless mass flux validate our assumption of the algorithms, i.e. Eq. (14). The profiles of the dimensionless temperature were also validated well, cf. Fig. $4 \mathrm{~b}$, even at the entrance sections of the channel. Figure 5 further compares the profiles of the average and centerline temperatures with different numbers of eigenfunctions. An overshooting at the entrance region occurs at very few items and gradually disappears with more items, anyway, even the computation as $N=1$ can predict well the main profiles due to the fast convergence of Whittaker functions. The overshooting at the entrance is because of the inconsistence between the inlet and wall boundary conditions, $T(0, y)=T_{\infty}$ and $T(x, D / 2)=T_{\mathrm{w}}$ at the top left corner $(x=0$ and $y$ $=D / 2)$. 

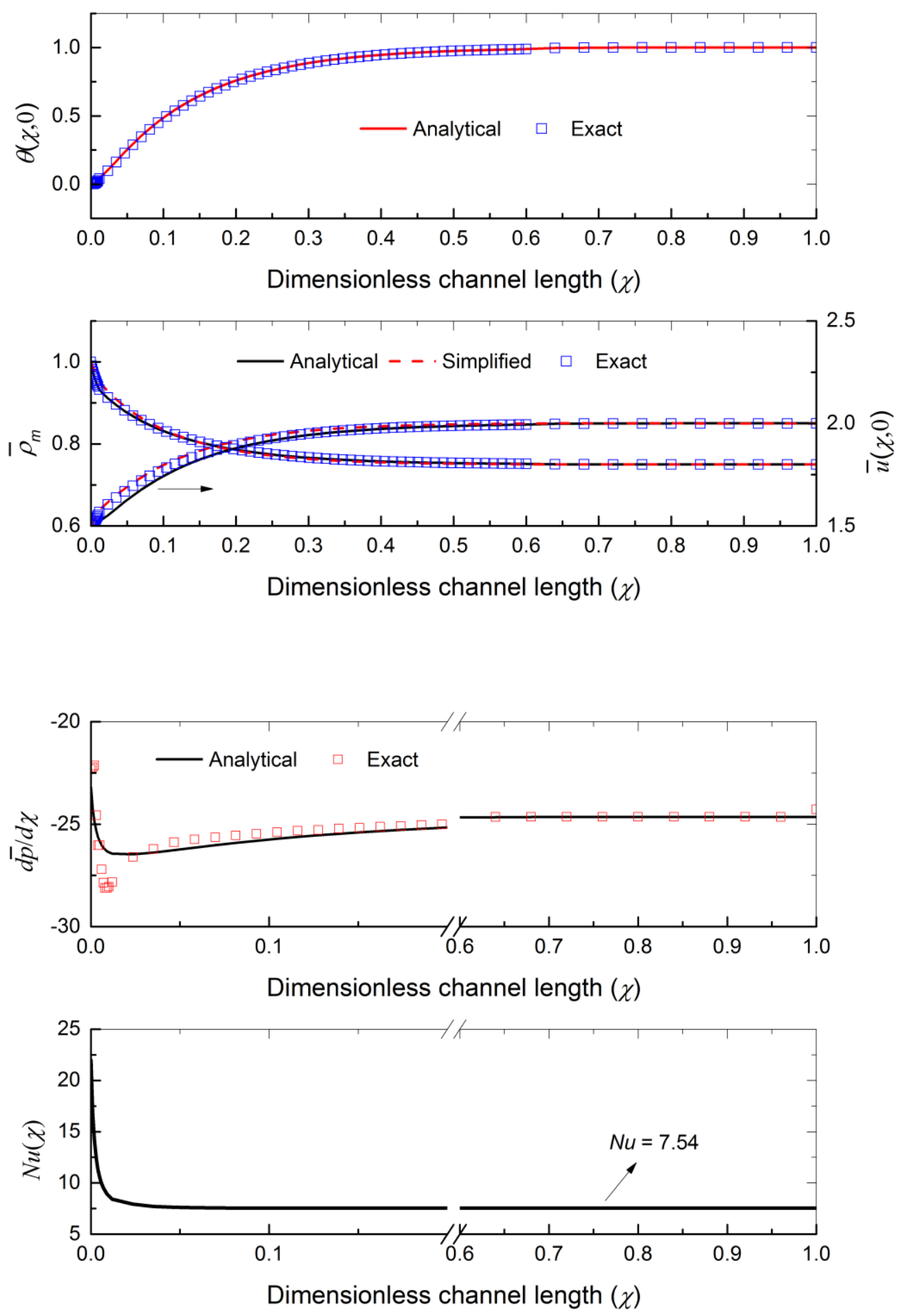

Fig. 3 Computational results for the constant WT case: (a) analytical and exact profiles of dimensionless temperature, density and velocity along the channel, (b) analytical and exact profiles of dimensionless pressure gradient (upper) and Nusselt number (lower). 

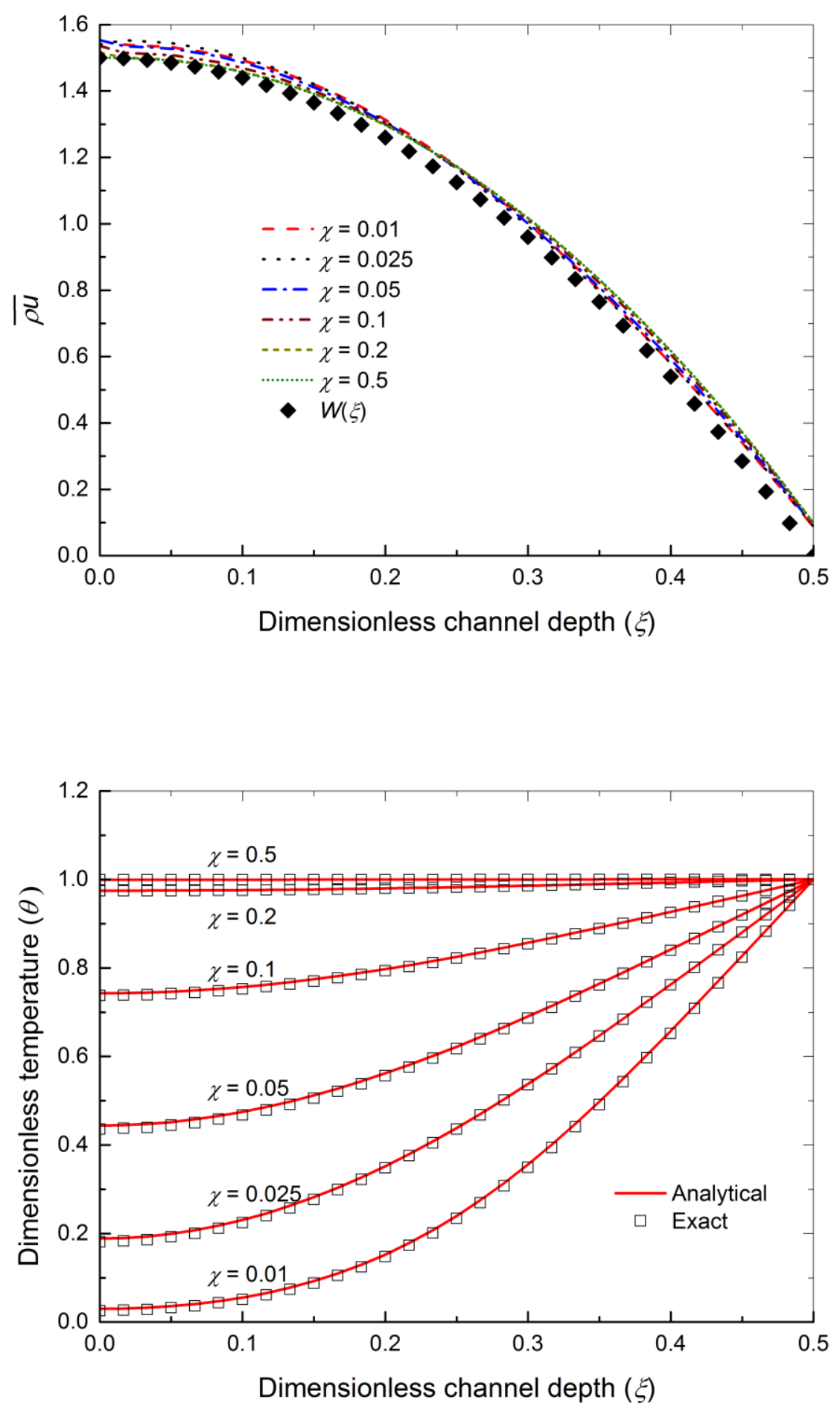

Fig. 4 Computational results for the constant WT case: profiles of (a) dimensionless mass flux and (b) dimensionless temperature, in the $y$ direction at different locations along the channel. 

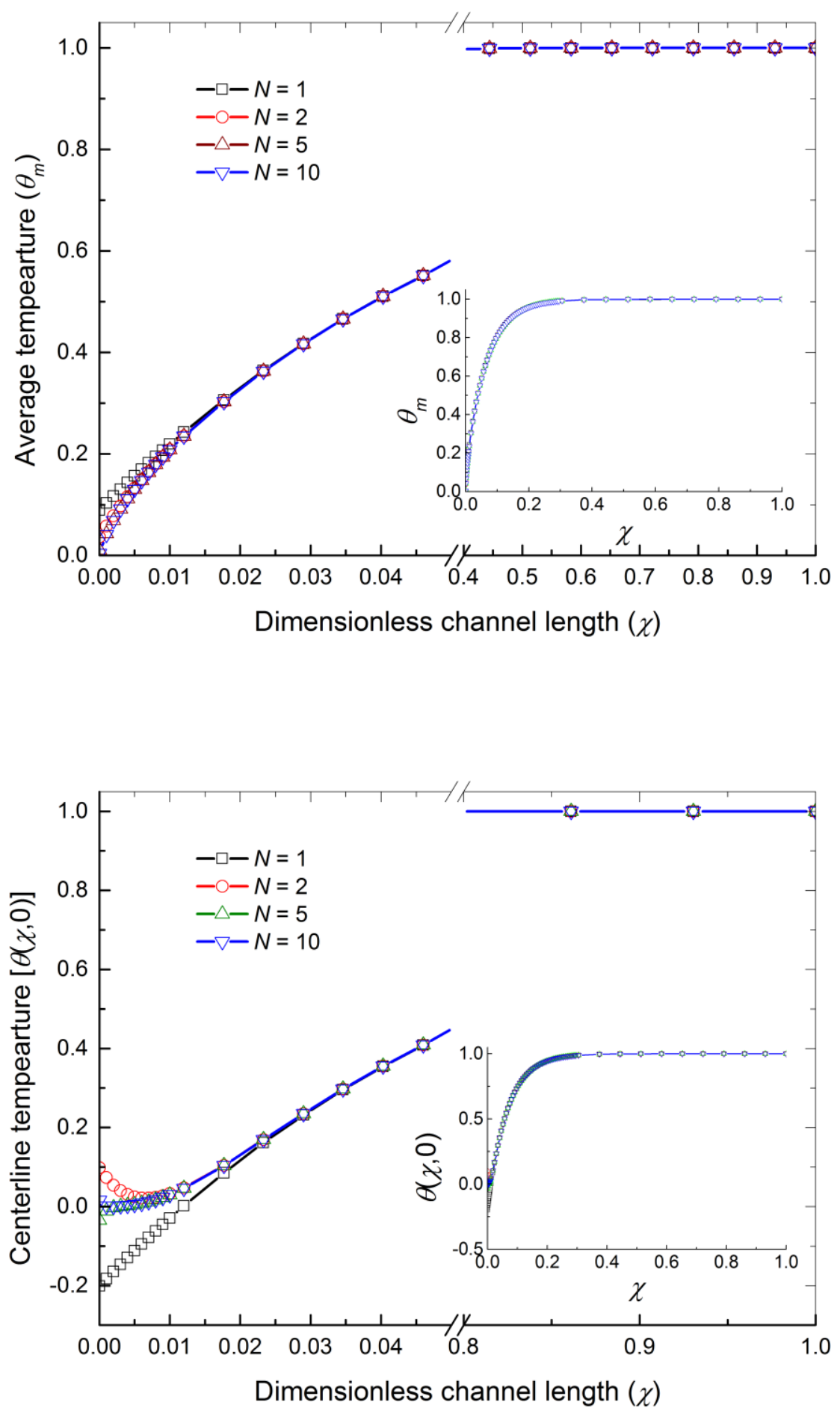

Fig. 5 Computational results for the constant WT case: profiles of (a) dimensionless average temperature and (b) dimensionless centerline temperature at different numbers of items.

Case 2: the distribution of the wall heat flux is in power-law form, i.e. 


$$
q(\chi)=\sum_{m=0}^{M} c_{m} \chi^{m}, \quad(\Delta T)_{0}=\frac{2 \gamma_{L D}}{\rho_{\infty} u_{\infty} c_{p}} \sum_{m=0}^{M} \frac{c_{m}}{m+1}
$$

Following Eq. (45) and Eq. (B3),

$$
\begin{aligned}
& \theta_{0}(\chi, \xi)=-\frac{D}{\kappa(\Delta T)_{0}}\left[\left(\xi^{2}-\sum_{n=0}^{N} a_{3 n} Y_{n}\right) \sum_{m=0}^{M} c_{m} \chi^{m}+3 b \sum_{m=0}^{M} \frac{c_{m}}{m+1} \chi^{m+1}\right]+\sum_{n=1}^{N} \varphi_{n} Y_{n}(\xi) \\
& \varphi_{n}(\chi)=-\frac{D}{\kappa(\Delta T)_{0}}\left(2 b a_{2 n}+a_{3 n} b_{n}\right) \sum_{m=0}^{M} c_{m}\left[\sum_{k=0}^{m} \frac{(-1)^{k}(m)_{k}}{\left(b_{n}\right)^{k+1}} \chi^{m-k}+\frac{(-1)^{m+1} m !}{\left(b_{n}\right)^{m+1}} e^{-b_{n} \chi}\right]
\end{aligned}
$$

Using Eq. (47), the Nusselt number is

$$
\begin{aligned}
& N u(\chi)=-\frac{d_{e}}{\kappa(\Delta T)_{0}\left[\theta_{0}\left(\chi, \frac{1}{2}\right)-\theta_{\mathrm{m}}(\chi)\right]} \sum_{m=0}^{M} c_{m} \chi^{m} \\
& \theta_{\mathrm{m}}(\chi)=-\frac{3 D b}{\kappa(\Delta T)_{0}} \sum_{m=0}^{M} \frac{c_{m}}{m+1} \chi^{m+1}
\end{aligned}
$$

Following Eq. (54), the pressure profile is

$$
\begin{gathered}
\frac{u_{\infty}^{2}}{2 R_{g}} \frac{d}{d \chi}\left[\left(\bar{p}+\frac{p_{\infty}}{\rho_{\infty} u_{\infty}^{2}}-\frac{54}{35 \beta_{\rho}}\right)^{2}\right]=-\frac{12 \gamma_{L D} \gamma_{d D} T_{\infty}}{\operatorname{Re}}+F(\chi) \\
+\frac{3 b D}{\kappa}\left(\frac{54}{35} \sum_{m=0}^{M} c_{m} \chi^{m}+\frac{12 \gamma_{L D} \gamma_{d D}}{\operatorname{Re}} \sum_{m=0}^{M} \frac{c_{m}}{m+1} \chi^{m+1}\right)-(\Delta T)_{0} \sum_{n=1}^{N} a_{4 n} \phi_{n}
\end{gathered}
$$

where

$$
\begin{aligned}
& F(\chi)=\frac{D}{\kappa}\left(\frac{3}{70}-\sum_{n=0}^{N} a_{3 n} a_{4 n}\right) \sum_{m=1}^{M} m c_{m} \chi^{m-1} \\
& \phi_{n}(\chi)=\frac{d \varphi_{n}}{d \chi}=-\frac{D}{\kappa(\Delta T)_{0}}\left(2 b a_{2 n}+a_{3 n} b_{n}\right) \times \\
& \sum_{m=0}^{M} c_{m}\left[\sum_{k=0}^{m-1} \frac{(-1)^{k}(m)_{k+1}}{\left(b_{n}\right)^{k+1}} \chi^{m-k-1}+\frac{(-1)^{m} m !}{\left(b_{n}\right)^{m}} e^{-b_{n} \chi}\right]
\end{aligned}
$$

Specially, for a constant wall heat flux, i.e. $q(\chi)=q_{0}\left(c=q_{0}\right.$ and $\left.m=0\right)$, there is

$$
\begin{gathered}
\theta_{0}(\chi, \xi)=-\frac{D q_{0}}{\kappa(\Delta T)_{0}}\left[\begin{array}{c}
\left(\xi^{2}-\sum_{n=0}^{N} a_{3 n} Y_{n}\right)+3 b \chi \\
\left.+\sum_{n=1}^{N}\left(\frac{2 b a_{2 n}}{b_{n}}+a_{3 n}\right)\left(1-e^{-b_{n} \chi}\right) Y_{n}(\xi)\right]
\end{array}\right. \\
N u(\chi)=-\frac{q(\chi) d_{e}}{\kappa(\Delta T)_{0}\left[\theta_{0}\left(\chi, \frac{1}{2}\right)-\theta_{\mathrm{m}}(\chi)\right]}, \quad \theta_{\mathrm{m}}(\chi)=-\frac{3 D b q_{0}}{\kappa(\Delta T)_{0}} \chi
\end{gathered}
$$




$$
\begin{aligned}
\frac{u_{\infty}^{2}}{2 R_{g}} \frac{d}{d \chi}\left[\left(\bar{p}+\frac{p_{\infty}}{\rho_{\infty} u_{\infty}^{2}}-\frac{54}{35 \beta_{\rho}}\right)^{2}\right]= & -\frac{12 \gamma_{L D} \gamma_{d D} T_{\infty}}{\operatorname{Re}}+\frac{3 b D q_{0}}{\kappa}\left(\frac{54}{35}+\frac{12 \gamma_{L D} \gamma_{d D}}{\operatorname{Re}} \chi\right) \\
& +\frac{D}{\kappa} q_{0} \sum_{n=1}^{N} a_{4 n}\left(2 b a_{2 n}+a_{3 n} b_{n}\right) e^{-b_{n} \chi}
\end{aligned}
$$

The pressure profile can be further simplified because of the rapid decay of the exponent items,

$$
\frac{d \bar{p}}{d \chi}=\frac{1}{1-\frac{54}{35 \beta_{\rho}} \gamma M a_{\infty}^{2}}\left[\frac{-12 \gamma_{L D} \gamma_{d D}}{\operatorname{Re}}+\frac{3 b D q_{0}}{\kappa T_{\infty}}\left(\frac{54}{35}+\frac{12 \gamma_{L D} \gamma_{d D}}{\operatorname{Re}} \chi\right)\right]
$$

Note that, the constant wall heat flux case shows a parabolic pressure profile, instead of a linear one. As a result, the centerline velocity is obtained by

$$
\bar{u}_{m}(\chi)=-\frac{35}{36 a_{0}^{2}}\left(\beta_{0} a_{0}-\beta_{1}\right)-\frac{35 \beta_{1}}{36 a_{0}} \chi+\left[\frac{3}{2}+\frac{35}{36 a_{0}^{2}}\left(\beta_{0} a_{0}-\beta_{1}\right)\right] e^{-a_{0} \chi}
$$

Figure 6 validated the analytical profiles of the dimensionless gas temperature at the centerline, the average density, centerline velocity, and pressure gradient along the channel in the constant WHF case. The parameters are employed as: $D=10^{-3} \mathrm{~m}, L=0.05 \mathrm{~m}, u_{\infty}=0.5 \mathrm{~m} \mathrm{~s}^{-1}, p_{\infty}=10^{5} \mathrm{~Pa}, q_{0}=$ $-600 \mathrm{~W} \mathrm{~m}^{-2},(\Delta T)_{0}=102.67 \mathrm{~K}$, which correspond to the dimensionless parameters as $\gamma_{L D}=50, \mathrm{Pr}$ $=0.744, R e=64.9, M a_{\infty}=0.0014, E c=2.419 \times 10^{-6}$ for air. The algorithms work well again. Instead of the linear pressure profile in the constant WT case, the linear pressure gradient denotes a parabolic profile of the gas pressure in the constant WHF case. Figure 7 further validates the profiles of the dimensionless mass flux and temperature. Without inconsistence between the boundary conditions, the close-form expansion with only two eigenfunctions $(N=1)$ works very well, and even only the direct-current items $(N=0)$ can predict well the main profiles, as shown in Fig. 8.
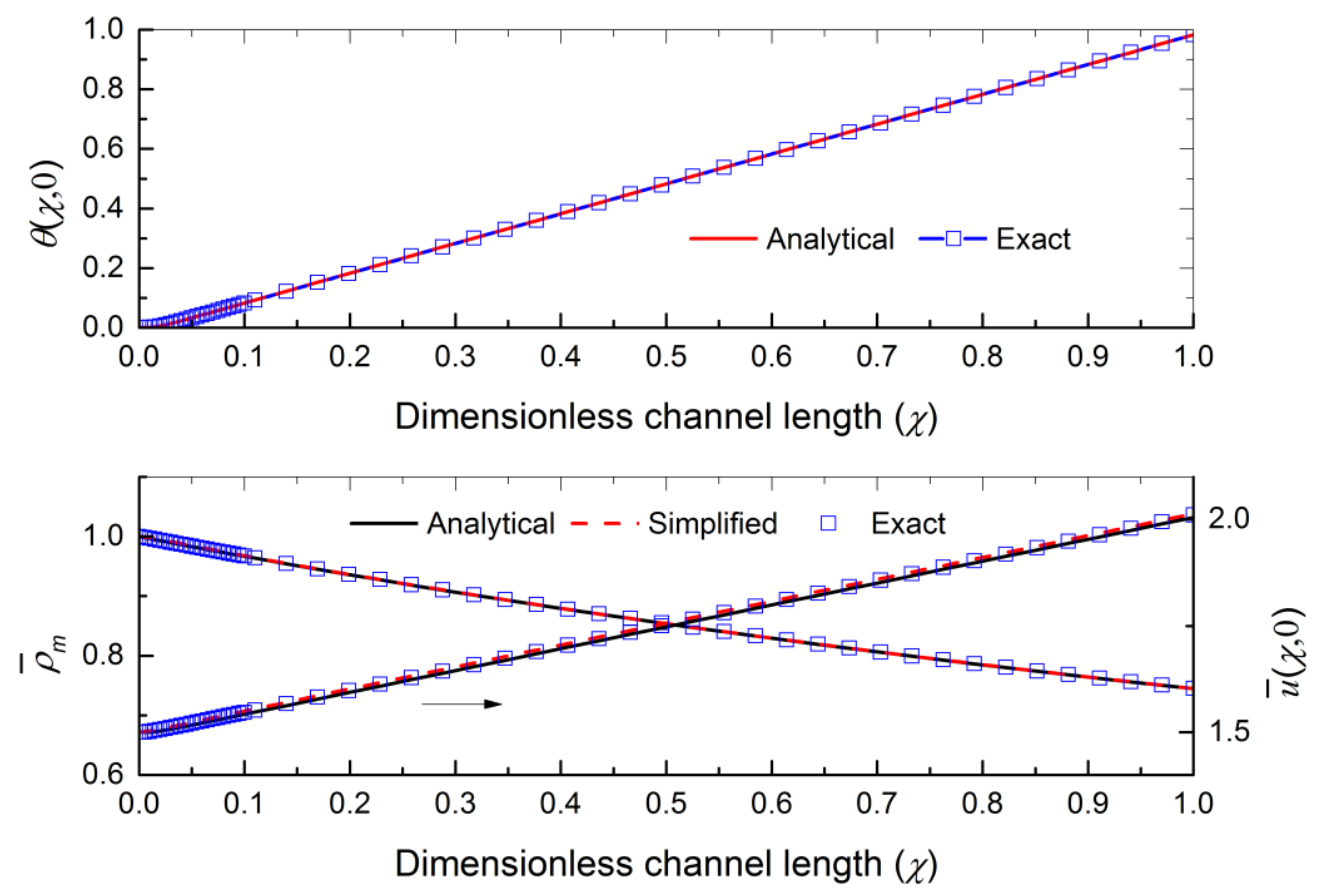

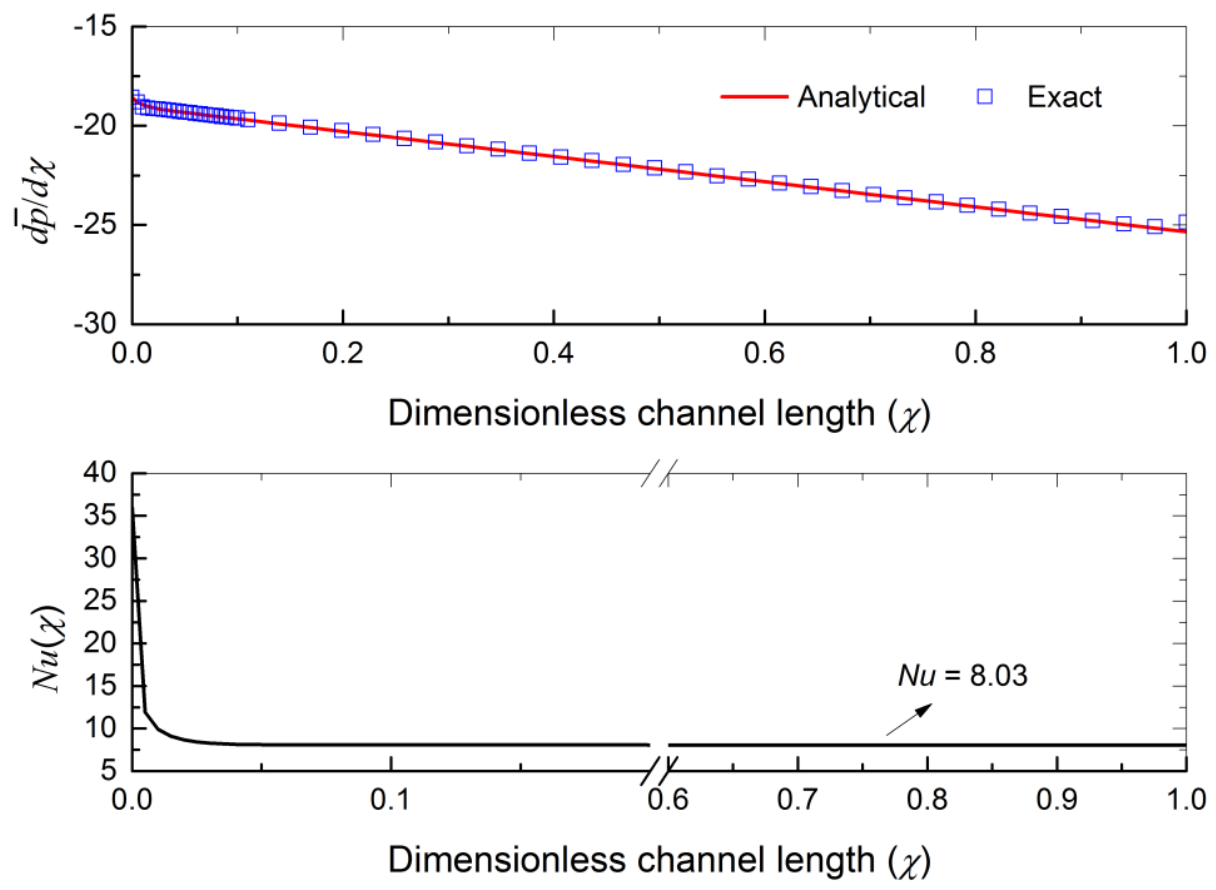

Fig. 6 Computational results for the constant WHF case: (a) analytical and exact profiles of dimensionless temperature, density and velocity along the channel, (b) analytical and exact profiles of dimensionless pressure gradient (upper) and Nusselt number (lower).

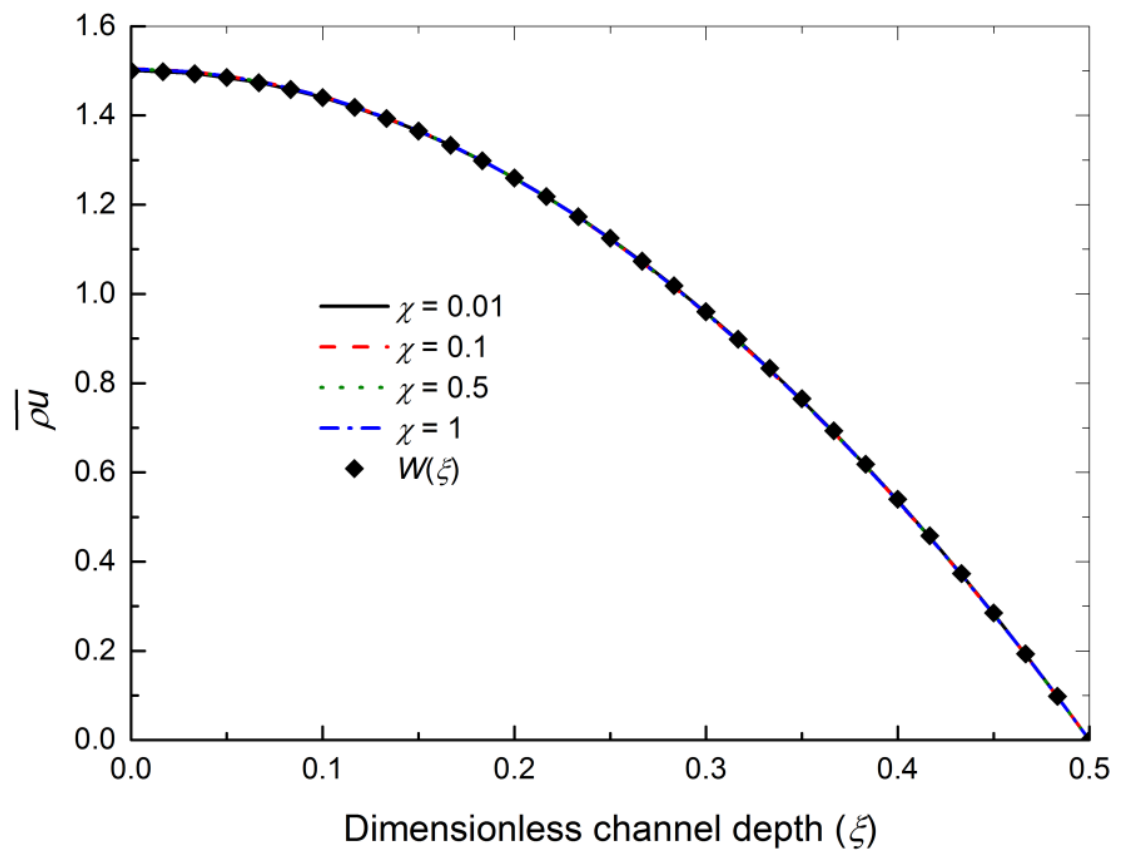




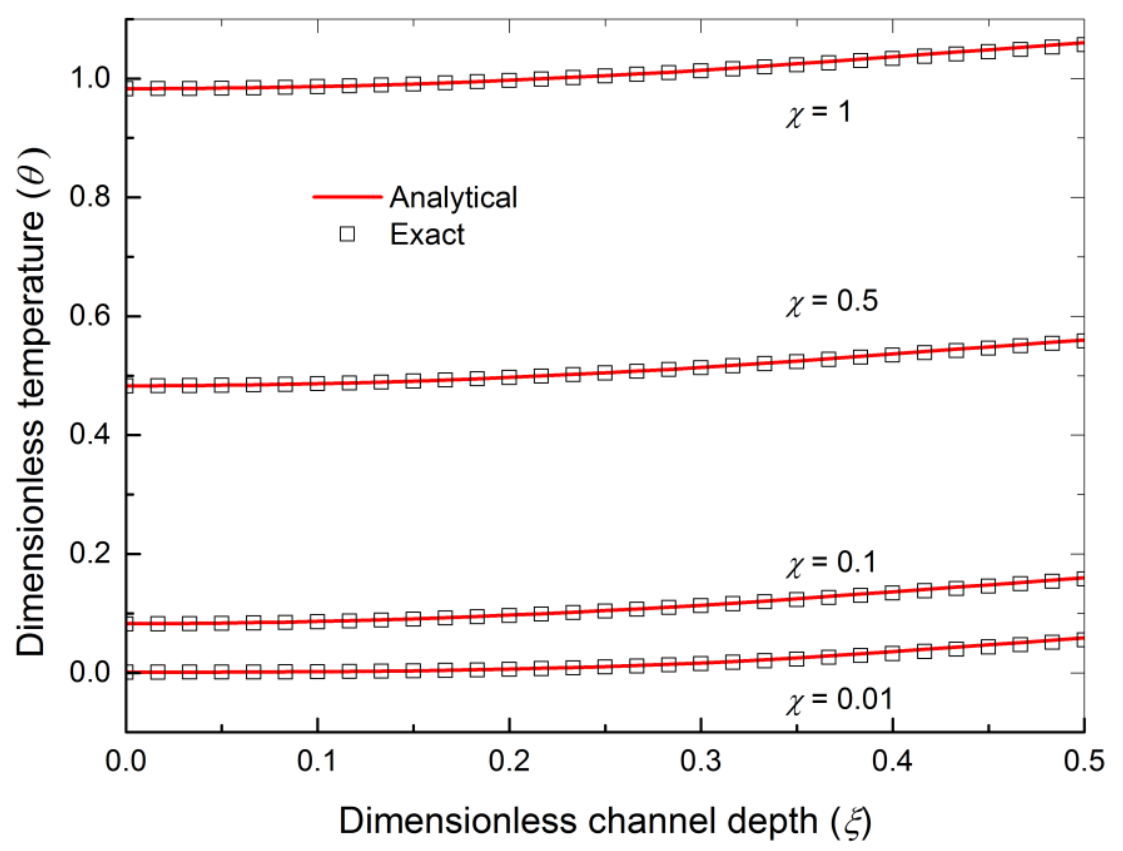

Fig. 7 Computational results for the constant WHF case: profiles of (a) dimensionless mass flux and (b) dimensionless temperature, in the $y$ direction at different locations along the channel.

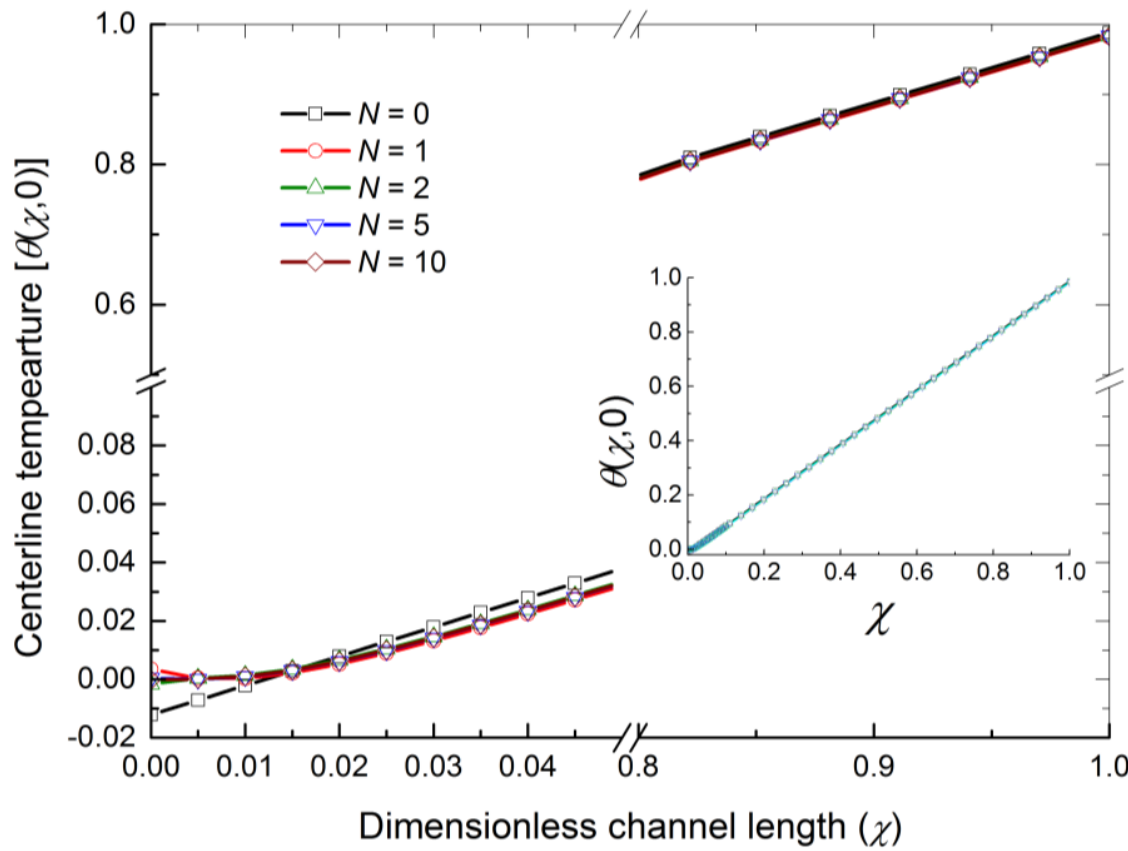

Fig. 8 Profiles of the dimensionless centerline temperature at different numbers of items in the constant WHF case. 
Case 3: The algorithms are finally validated at moderate $R e$ and $M a_{\infty}$ (i.e. $E c$ ). The parameters are designed as $D=10^{-4} \mathrm{~m}, L=5 \times 10^{-3} \mathrm{~m}, u_{\infty}=100 \mathrm{~m} \mathrm{~s}^{-1}, p_{\infty}=1.146 \times 10^{5} \mathrm{~Pa}$ (correspondingly the outlet pressure of $\left.10^{5} \mathrm{~Pa}\right), q(\chi)=-1.4026 \times 10^{5}\left(\chi^{2}-0.5 \chi\right) \mathrm{W} \mathrm{m}^{-2},(\Delta T)_{0}=8.726 \mathrm{~K}$, which correspond to the dimensionless parameters as $\gamma_{L D}=50, R e=1487.5, M a_{\infty}=0.288, E c=1.139$ for air.

Figure 9a shows the zero-order and first-order solutions of the dimensionless centerline temperature along the channel. Corresponding to the small value of the overall temperature rise, $(\Delta T)_{0}$, the heat transfer between gas and wall is overwhelmed by the dissipation effect due to the high gas velocity. Therefore, the first-order solution dominates the temperature profile. Here $q(\chi)>$ 0 means heat flows from the gas to the wall and vice versa. It is natural that the gas is cooled at the first half of the channel as $q(\chi)>0$. In the region of $\chi=0.5 \sim 0.9$, the gas is heated by the wall as $q(\chi)$ $<0$, however, the centerline temperature gradually decreases because of the stronger thermal effect of expansion. Figure $9 \mathrm{a}$ and $9 \mathrm{~b}$ further shows the analytical and exact along-the-channel profiles of the average gas temperature, wall temperature, gas velocity and pressure gradient. The algorithms work well again in this case of moderate Reynolds number and Mach number.

\section{Conclusions}

The classical problem of compressible laminar thermal flow in a flat mini- or micro-channel was revisited in this article. The hydrodynamic equations were introduced following two-dimensional continuum flow model, reduced to a system similar to the boundary-layer equations accounting for the geometric characteristics of mini- or micro-channels (large length/depth ratio), and then normalized related to dimensionless quantities including length/depth ratio $\left(\gamma_{L D}\right)$, Reynolds number $(R e)$, Prandtl number $(P r)$, Mach number $\left(M a_{\infty}\right)$ and Eckert number $(E c)$. Considering the much faster development of velocity than that of temperature, the momentum equation was decoupled with the energy equation by assuming that the mass flux (or velocity for simplification in cases of small/moderate temperature rise) holds a parabolic profile like the velocity profile of an incompressible parallel flow in a straight channel. Then analytical solutions of the dimensionless model were achieved in closed-form symbolic algebras of Whittaker eigenfunctions, corresponding to two kinds of boundary conditions with arbitrarily prescribed wall temperature or wall heat flux. As the eigenvalues and eigenfunctions (in $y$ direction) are independent on the dimensionless quantities, which influence the along-the-channel behaviors, our algorithm reveals the common features of all kinds of compressible laminar thermal flows. The algorithm is robust without assuming the linear distribution of pressure. Then the algorithm was applied to two specific cases with the prescribed wall temperature or wall heat flux in power-law profile. The analytical solutions, both the zero-order and first-order parts corresponding to the perturbation variable of $E c$, were exemplarily validated well via comparison with the exact (numerical) solutions, in the cases of small $M a_{\infty}$ or $E c$ with boundary conditions of constant wall temperature or constant wall heat flux, and in the case of moderate $R e$ and $M a_{\infty}$ (or $E c$ ) with boundary condition of parabolic wall heat flux.

This work is helpful to understand heat transfer in compressible laminar flow in mini- or microchannels, a general problem in fields of fuel cells, electronics, micro heat exchangers, etc. Analytical solution including the effects of gas injection/suction will be discussed in the next. 

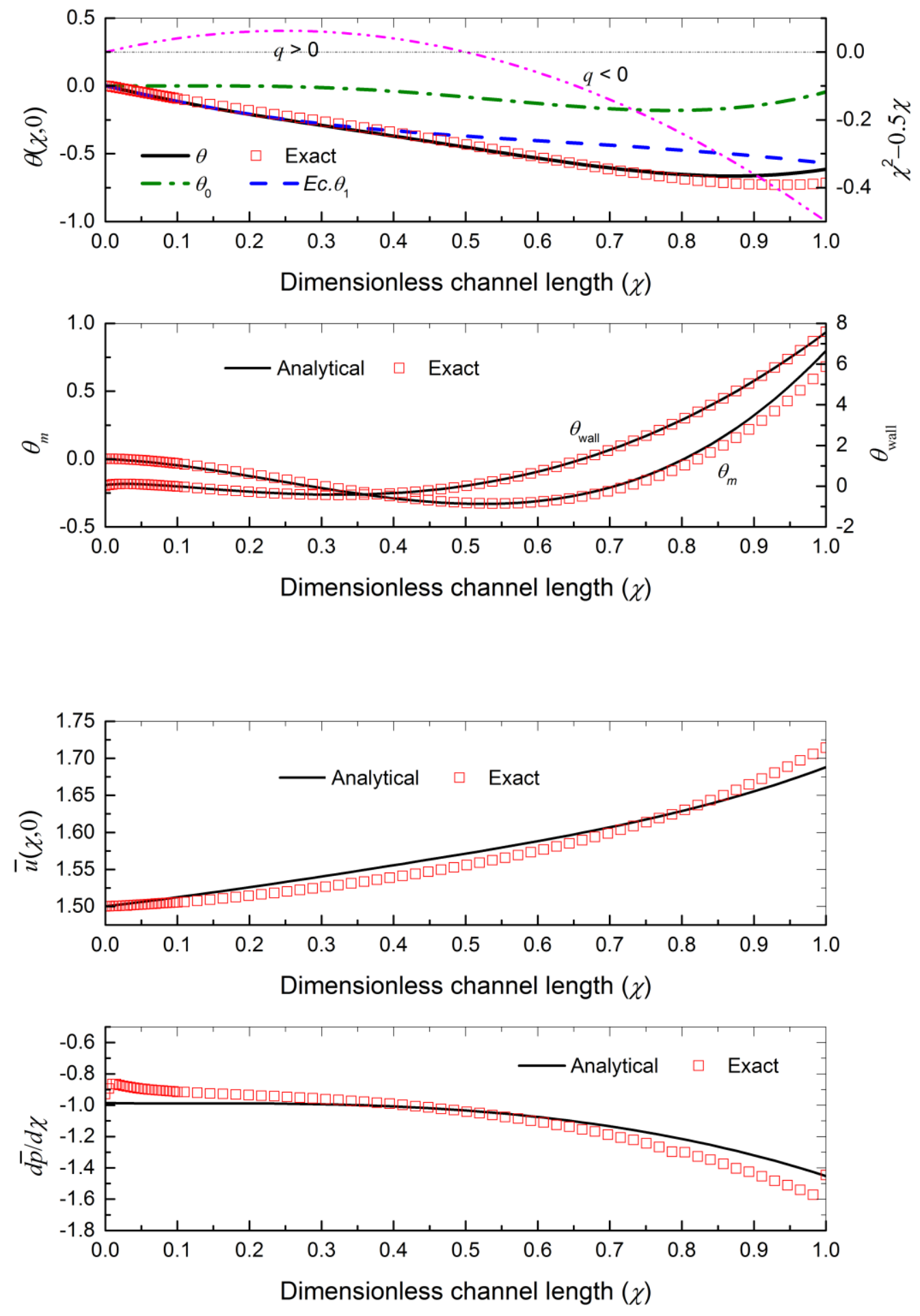

Fig. 9 Comparison between the analytical and exact solutions at moderate Reynolds number and Mach number with along-the-channel profiles of (a) zero- and first-order centerline gas temperature, (b) average gas temperature and wall temperature, (c) gas centerline velocity and (d) pressure gradient.

\section{Appendix A: Whittaker function and its properties}


The standard solutions of Whittaker's equation, $M_{\kappa, \mu}(z)$ and $W_{\kappa, \mu}(z)$ are [17]

$$
M_{\kappa, \mu}(z)=e^{-\frac{1}{2} z} z^{\frac{1}{2}+\mu} \sum_{s=0}^{\infty} \frac{\left(\frac{1}{2}+\mu-\kappa\right)_{s}}{(1+2 \mu)_{s} s !} z^{s}
$$

When $2 \mu$ is not an integer,

$$
W_{\kappa, \mu}(z)=\frac{\Gamma(-2 \mu)}{\Gamma\left(\frac{1}{2}-\mu-\kappa\right)} M_{\kappa, \mu}(z)+\frac{\Gamma(2 \mu)}{\Gamma\left(\frac{1}{2}+\mu-\kappa\right)} M_{\kappa,-\mu}(z)
$$

where $\Gamma(\cdot)$ is the gamma function.

When $z \rightarrow 0$, there is limiting form

$$
M_{\kappa, \mu}(z)=z^{\frac{1}{2}+\mu}(1+O(z)), \quad 2 \mu \neq-1,-2,-3, \ldots
$$

The differential of $M_{\kappa, \mu}(z)$ is

$$
\frac{d M_{\kappa, \mu}(z)}{d z}=\left(\kappa+\mu+\frac{1}{2}\right) z^{-1} M_{\kappa+1, \mu}(z)-\left(\kappa z^{-1}-\frac{1}{2}\right) M_{\kappa, \mu}(z)
$$

\section{Appendix B: Integral of $e^{-b x} \int e^{b \tau} \tau^{m} d \tau$}

Making the integral

$$
F_{m}(x)=\int_{0}^{x} e^{b \tau} \tau^{m} d \tau \quad(b \neq 0)
$$

Using integration by parts, it is easy to get

$$
\left\{\begin{array}{l}
F_{m}(x)=\frac{1}{b}\left[e^{b x} x^{m}-m F_{m-1}(x)\right] \quad(m \geq 1), \\
F_{0}(x)=\frac{1}{b}\left(e^{b x}-1\right)
\end{array}\right.
$$

As a result of the above the recurrence relation,

$$
e^{-b x} F_{m}(x)=(-1)^{m+1} \frac{m !}{b^{m+1}} e^{-b x}+\sum_{k=0}^{m}(-1)^{k} \frac{(m)_{k}}{b^{k+1}} x^{m-k}
$$

where

$$
(m)_{k}=m(m-1) \ldots \ldots(m-k+1), \quad(m)_{0}=1
$$

\section{Acknowledgments}

This work was supported by the Beijing Science and Technology Project [grant number Z181100004518004] and the Fundamental Research Funds for the Central Universities [grant number FRF-GF-17-B31]. CB thanks for the China Scholarship Council (CSC) fellowship support. $\mathrm{CB}$ also thanks Dr. Ruifeng Dou and Prof. Xiangjun Liu for helpful discussions.

\section{References}

[1]S.G. Kandlikar, S. Garimella, D. Li, S. Colin, M.R. King, Heat Transfer and fluid flow in 
minichannels and microchannels. Elsevier (2014).

[2]J. Sarkar, A critical review on convective heat transfer correlations of nanofluids, Renew Sust Energ Rev, 15(6) (2011) 3271-3277.

[3]T. Dixit, I. Ghosh, Review of micro- and mini-channel heat sinks and heat exchangers for single phase fluids, Renew Sust Energ Rev, 41 (2015) 1298-1311.

[4]A.A. Hussien, M.Z. Abdullah, M.A. Al-Nimr, Single-phase heat transfer enhancement in micro/minichannels using nanofluids: Theory and applications, Appl Energ, 164 (2016) 733-755.

[5]M.M. Awad, A.S. Dalkilic, S. Wongwises, A critical review on condensation heat transfer in microchannels and minichannels. J Nanotechnol Eng Med, 5(1) (2014) 010904-010904-25.

[6]H.C. Brinkman, Heat Effects in Capillary Flow-I, Appl Sci Res, 2(2) (1950) 120-124.

[7]R. Siegel, E.M. Sparrow, T.M. Hallman, Steady laminar heat transfer in a circular tube with prescribed wall heat flux, Appl Sci Res, 7(5) (1958) 386-392.

[8]R.F. Barron, X.M. Wang, T.A. Ameel, R.O. Warrington, The Graetz problem extended to slipflow, Int J Heat Mass Tran, 40(8) (1997) 1817-1823.

[9]N.G. Hadjiconstantinou, O. Simek, Constant-wall-temperature Nusselt number in micro and nano-channels, J Heat Trans-T Asme, 124(2) (2002) 356-364.

[10]Y. Rouizi, D. Maillet, Y. Jannot, Fluid temperature distribution inside a flat mini-channel: Semianalytical wall transfer functions and estimation from temperatures of external faces, Int J Heat Mass Tran, 64 (2013) 331-342.

[11]R.K. Prudhomme, T.W. Chapman, J.R. Bowen, Laminar compressible flow in a tube, Appl Sci Res, 43(1) (1986) 67-74.

[12]H.R. Vandenberg, C.A. Tenseldam, P.S. Vandergulik, Compressible laminar-flow in a capillary, J Fluid Mech, 246 (1993) 1-20.

[13]H.R. Vandenberg, C.A. Tenseldam, P.S. Vandergulik, Thermal effects in compressible viscousflow in a capillary, Int J Thermophys, 14(4) (1993) 865-892.

[14]J.C. Harley, Y.F. Huang, H.H. Bau, J.N. Zemel, Gas-flow in micro-channels, J Fluid Mech, 284 (1995) 257-274.

[15]H. Schlichting, K. Gersten, Boundary-layer theory, $9^{\text {th }}$ edition, Berlin: Springer, (2016).

[16]Z.Y. Guo, X.B. Wu, Further study on compressibility effects on the gas flow and heat transfer in a microtube, Microscale Therm Eng, 2(2) (1998) 111-120.

[17]F.W.J. Olver, D.W. Lozier, R.F. Boisvert, C.W. Clark, NIST handbook of mathematical functions, Cambridge (2010).

[18]A. Aubert, F. Candelier, C. Solliec, Semi-analytical solution for heat transfer in a water film flowing over a heated plane, J Heat Trans-T ASME, 132(6) (2010) 064501.

[19]A.E. Quintero, M. Vera, Laminar counterflow parallel-plate heat exchangers: An exact solution including axial and transverse wall conduction effects, Int J Heat Mass Tran, 104 (2017) 12291245.

[20]COMSOL Multiphysics, User's Guide Version 3.5a. 